\title{
Quasiguided modes and optical properties of photonic crystal slabs
}

\author{
S. G. Tikhodeev, ${ }^{1}$ A. L. Yablonskii, ${ }^{1}$ E. A. Muljarov, ${ }^{1,2}$ N. A. Gippius,${ }^{1,2}$ and Teruya Ishihara ${ }^{2}$ \\ ${ }^{1}$ General Physics Institute RAS, Vavilova 38, Moscow 119991, Russia \\ ${ }^{2}$ Institute of Physical and Chemical Research (RIKEN), Wako 351-0198, Japan
}

(Received 12 February 2002; published 8 July 2002)

\begin{abstract}
We formulate a scattering-matrix-based numerical method to calculate the optical transmission properties and quasiguided eigenmodes in a two-dimensionally periodic photonic crystal slab (PCS) of finite thickness. The square symmetry (point group $C_{4 v}$ ) is taken for the illustration of the method, but it is quite general and works for any point group symmetry for one-dimensional (1D) and 2D PCS's. We show that the appearance of well-pronounced dips in the transmission spectra of a PCS is due to the interaction with resonant waveguide eigenmodes in the slab. The energy position and width of the dips in transmission provide information on the frequency and inverse radiative lifetime of the quasiguided eigenmodes. We calculate the energies, linewidths, and electromagnetic fields of such quasiguided eigenmodes, and analyze their symmetry and optical activity. The electromagnetic field in such modes is resonantly enhanced, which opens possibilities for use in creating resonant enhancement of different nonlinear effects.
\end{abstract}

DOI: 10.1103/PhysRevB.66.045102

PACS number(s): 42.70.Qs, 78.66.-w, 73.20.Mf

\section{INTRODUCTION}

A considerable effort has been devoted in recent years to the investigation of finite-thickness slabs of photonic crystals. ${ }^{1-12}$ Such photonic crystal slabs (PCS's), especially if they support waveguide modes, demonstrate various interesting properties aimed at active photonic devices for future optical communication networks. For example, the transmission spectra of waveguiding PCS's have well-pronounced narrow dips which exhibit a complicated behavior with the change of angle of light incidence and of the geometrical parameters of the system.

Such features in the transmission spectra of gratings are called Wood's anomalies; see, e.g., Ref. 13. Generally, there are two types of Wood's anomalies: diffractive anomalies, caused by openings of the new diffraction orders (diffraction thresholds), and waveguiding anomalies, caused by a resonant interaction of the incoming light with surface or waveguide modes. Historically, diffractive-type anomalies were first found in metallic shallow gratings by Wood, ${ }^{14}$ and theoretically explained by Rayleigh. ${ }^{15}$ They are also called Rayleigh anomalies. Although both types of anomalies appear in the spectrum of PCS's, waveguiding anomalies demonstrate a richer behavior. Basically speaking, waveguiding anomalies are examples of a Fano resonance, ${ }^{16}$ where a discrete mode becomes a resonance due to the interaction with continua. In this paper we mainly focus on such anomalies in waveguiding PCS's.

Recently we developed a scattering matrix numerical method, ${ }^{17-19}$ based on a generalization of the method proposed by Whittaker and Culshaw, ${ }^{7}$ to model the properties of multilayered one-dimensional and two-dimensional patterned PCS's with frequency dispersive constituent materials. Our motivation was to explain the experimentally measured $^{20,18}$ transmission spectra in PCS's containing an inorganic-organic semiconductor imbedded into a $1 \mathrm{D}$ or $2 \mathrm{D}$ periodically patterned quartz substrate. The narrow dips in transmission are due to the excitation of quasiguided (or leaky) modes, which appear to play a crucial role in the optical properties of waveguiding PCS.

The goal of the present paper is to develop a method to calculate the energies, linewidths (inverse lifetimes), and electromagnetic field distributions of photonic eigenmodes in periodically patterned photonic crystals of finite thickness, and to analyze the symmetry of such modes and their optical activity. Basically, photonic crystal slabs are periodically corrugated waveguides or grating-waveguide structures. During the last years, the basic understanding of quasiquided modes nature in such structures has been developed (see, e.g., Refs. 13 and 21). A limited analysis of the leaky modes in PCS's was obtained via the finite-difference time-domain method; see Ref. 22, and references therein. However, until recently there have been no direct methods developed to calculate the energy and linewidth dispersion as well as the electromagnetic field distribution of quasiguided modes in arbitrary PCS's. Vector-field ${ }^{9}$ and perturbative ${ }^{10,11}$ models were been proposed recently, but are valid only for thin PCS's.

On the other hand, a powerful scattering matrix formalism ${ }^{23}$ allows a full description of such modes in arbitrary PCS's. The scattering matrix formalism is utilized instead of a more familiar transfer-matrix formalism. The former provides an advantage in such a periodic structure in that evanescent waves are inevitably involved for higher reciprocal-lattice vectors. To the best of our knowledge, the first example of the use of this powerful formalism to calculate transmissivity, reflectivity and light emission of arbitrary thick PCS on a substrate was carried out by Whittaker and Culshaw ${ }^{7}$ and by Wendler and Kraft, ${ }^{8}$ (also see Ref. 24) but there was no direct calculation of the eigenmodes provided. Recently the scattering matrix method was used to calculate the eigenmodes in a free-suspended PCS's, ${ }^{12}$ but only below the vacuum cone where such modes retain a purely guided character. But the properties of quasi-guided modes in arbitrary PCS on a substrate have, to the best of our knowledge, not yet been calculated.

Following Ref. 25, we use another scheme based on the electric-field-resolved Maxwell equations in the constituent 
layers (instead of the magnetic-field-resolved equations shown in Ref. 7) which appears to be quite optimized, at least for the dielectric structures. Although the preliminary results of our method in comparison with experiments for 1D and $2 \mathrm{D}$ periodic photonic crystal slabs with a polariton pole were already reported, ${ }^{17-19}$ we have not yet published the details of our calculation scheme. The inclusion of a polariton pole manifests itself strongly in the optical properties of the PCS. The number of experimentally discernible features (Wood's anomalies) in the optical spectra increases, and their dispersion becomes more complicated due to the polariton effect. However, a basic understanding of the physics of the PCS optical response such as the origin of the dips in the transmissivity spectra and their connection with the PCS photon eigenmodes, can be acquired more easily by a simplified example omitting the dispersion. Thus, in order to simplify the discussion, in this paper we restrict ourselves to a PCS with constant local dielectric susceptibilities of all constituent materials.

The structure of the paper is as follows. In Sec. II we describe the model structure (a square-symmetry dielectric PCS on a substrate) and the geometry of the light incidence and its interaction with Bragg harmonics, and introduce quasiguided modes in PCS's, using an empty lattice approximation. In Sec. III the formulation of the scattering matrix method is given (Sec. III A), and illustrated on the example of the model structure (Sec. III B). In Sec. IV the scatteringmatrix method is introduced to calculate the properties of quasiguided modes in PCS's (Sec. IV A) and illustrated using a model structure (Sec. IV B). All technical details of the formulation are furnished in the Appendixes $A-G$, which are self-contained, give a full formulation of the scattering matrix method, and can be read independently of the main text.

\section{ESSENTIALS OF LIGHT INTERACTION WITH A PHOTONIC CRYSTAL SLAB}

\section{A. Model structure and geometry of light incidence}

A typical structure of interest (the model structure hereafter) and the geometry of $S$ - and $P$-polarized light incidence is illustrated in Fig. 1. We use the orthogonal axes $(\hat{\mathbf{x}}, \hat{\mathbf{y}}, \hat{\mathbf{z}})$ as shown in this figure. The positive direction of the $\hat{\mathbf{z}}$ axis is set from the vacuum into the substrate. The polar and azimuthal angles of incidence $\vartheta$ and $\varphi$ specify the incoming light incidence. The azimuthal angle $\varphi$ is measured between the $x$ axis and the plane of light incidence; $\varphi=0$ in Fig. 1.

Hereafter, for numerical calculations we assume the substrate material to be quartz (substrate dielectric constant $\varepsilon_{\mathrm{s}}$ $=2.132$ ), and the higher dielectric constant active material to be a semiconductor with $\varepsilon_{\infty} \sim 3.97$ (which corresponds to the background dielectric constant of the self-organized inorganic-organic semiconductor in Refs. 20 and 18).

The symmetry of the 2D periodicity of the model structure is taken to be square (point group $C_{4 v}$ ), but the method itself is quite general and can be readily applied for any point group symmetry. The in-plane periods are taken as $d_{x}=d_{y}$ $=d \sim 680 \mathrm{~nm}$, and the vertical thickness of the patterned layer is $L_{z} \sim 120 \mathrm{~nm}$. The ratio of the in-plane size of the

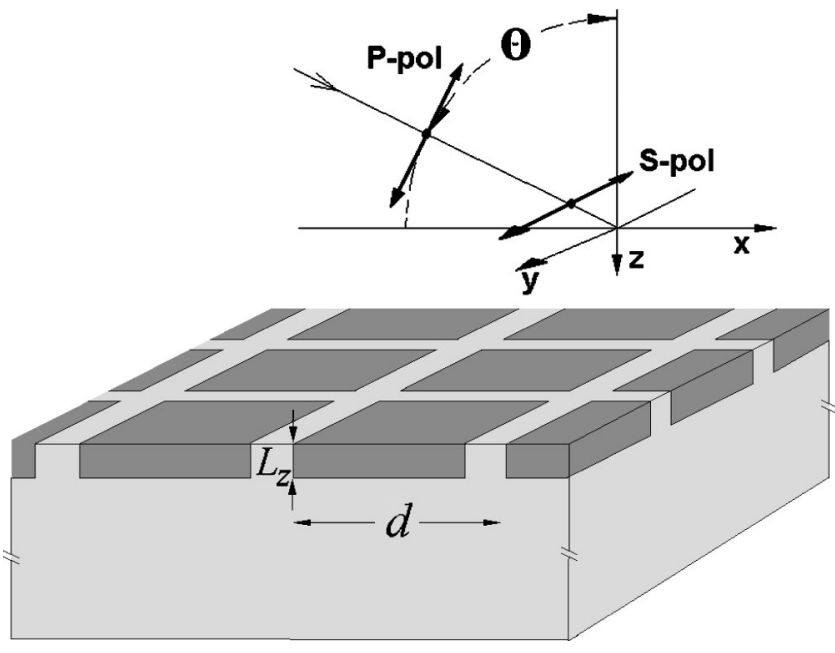

FIG. 1. Schematic structure of a square-patterned guiding layer on a substrate. Light gray: substrate (e.g., quartz); dark gray: optically active material with a higher dielectric constant. The coordinate origin $z=0$ is at the surface of the structure.

semiconductor squares to the thickness of quartz walls is taken $\eta \sim 4$. These values are close to those characteristic for the experiments ${ }^{20,26,18}$ with inorganic-organic semiconductor superlattices imbedded into a patterned quartz substrate, but neglecting the polariton dispersion of the active material.

The 2D periodic slab couples an incoming light of frequency $\omega$ and wave vector $\mathbf{k}=\left(k_{x}, k_{y}, k_{z}\right)$,

$$
\begin{gathered}
k_{x}=\frac{\omega}{c} \sin \vartheta \cos \varphi, \quad k_{y}=\frac{\omega}{c} \sin \vartheta \sin \varphi, \\
k_{z}=\frac{\omega}{c} \cos \vartheta,
\end{gathered}
$$

with all Bragg harmonics of the same frequency $\omega$ and wave vectors

$$
\mathbf{k}_{\mathbf{G}, a}^{ \pm}=\left(k_{x, \mathbf{G}}, k_{y, \mathbf{G}}, \pm k_{z, \mathbf{G}, a}\right),
$$

where $^{27}$

$$
\begin{gathered}
k_{x, \mathbf{G}}=k_{x}+G_{x}, \quad k_{y, \mathbf{G}}=k_{y}+G_{y}, \\
k_{z, \mathbf{G}, a}=\sqrt{\frac{\omega^{2} \varepsilon_{a}}{c^{2}}-\left(k_{x}+G_{x}\right)^{2}-\left(k_{y}+G_{y}\right)^{2}},
\end{gathered}
$$

$a=\mathrm{v}$ for vacuum $\left(\varepsilon_{\mathrm{v}}=1\right)$ and $a=\mathrm{s}$ for the substrate, and

$$
\mathbf{G}=\frac{2 \pi}{d}\left(g_{x}, g_{y}, 0\right), g_{x, y}=0, \pm 1, \pm 2, \ldots
$$

is the $2 \mathrm{D}$ reciprocal square lattice.

In this paper we assume the substrate to be optically transparent $\left(\operatorname{Im} \varepsilon_{\mathrm{s}}=0\right)$. Thus, depending on the incoming light frequency $\omega$ (real number) the Bragg harmonics [Eq. (3)] are either propagating or exponential. Hereinafter we refer to the harmonics, exponentially growing (decreasing) away from the PCS's as increscent (evanescent) harmonics. If $k_{z, \mathbf{G}, a}^{2}$ $>0, a=\mathrm{v}$ and $\mathrm{s}$, the corresponding harmonics are purely 


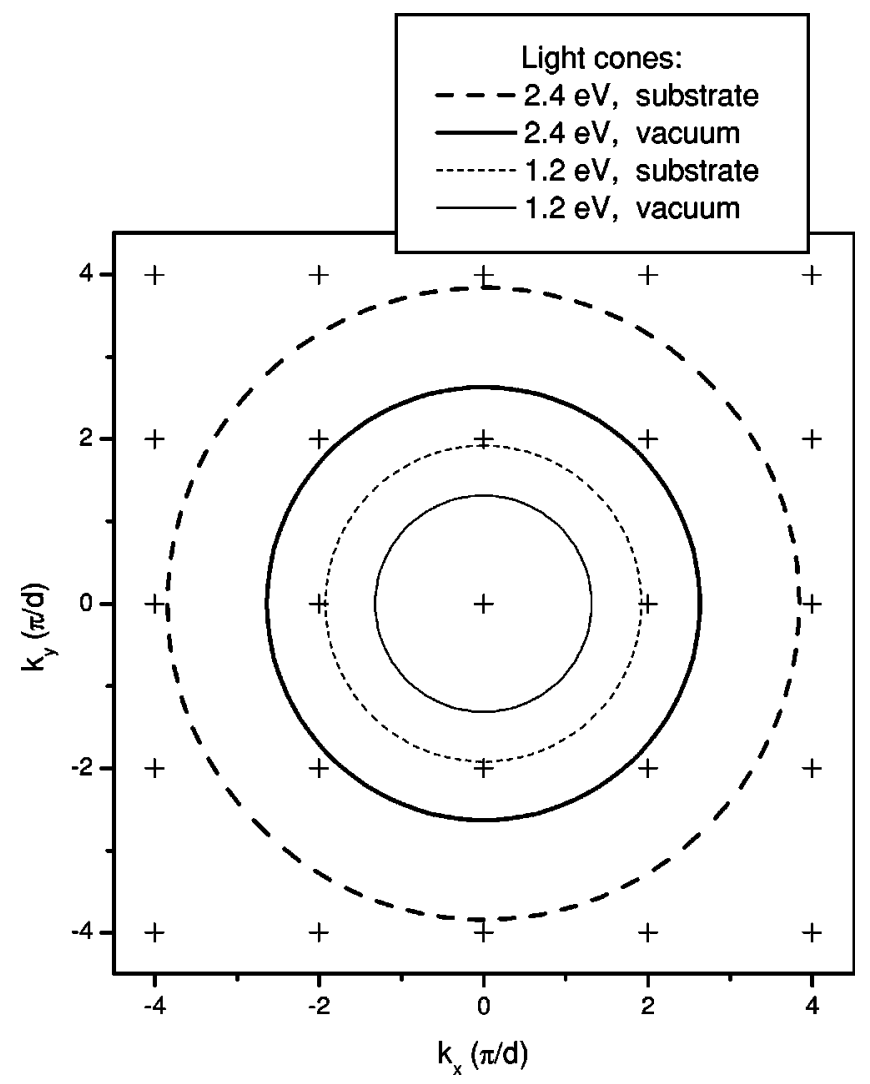

FIG. 2. The map of Bragg harmonics $\left(k_{x, \mathbf{G}}, k_{y, \mathbf{G}}\right),[\mathrm{Eq}$. (4)] of the $k_{x}=k_{y}=0$ incoming light (pluses). Solid and dashed circles show the vacuum and substrate light cone cross sections for $\omega$ $=1200$ and $2400 \mathrm{meV}$ (thin and thick lines, respectively). For each $\omega$, the harmonics inside the solid circle are radiative modes both in vacuum and substrate, and those between solid and dashed circles are exponential partial waves in vacuum and radiative modes in substrate. The harmonics outside both cones are the exponential modes.

propagating in both the vacuum and substrate. If $k_{z, \mathbf{G}, \mathrm{v}}^{2}<0$ and $k_{z, \mathbf{G}, \mathbf{s}}^{2}>0$, the harmonics are propagating in vacuum and exponential in the substrate. If $k_{z, \mathbf{G}, a}^{2}<0, a=\mathrm{v}$ and $\mathrm{s}$, the corresponding harmonics are exponential in both the vacuum and substrate. Here we suppose that $(z, t)$ dependence of the field in each harmonic is proportional to $\exp \left( \pm i k_{z, \mathbf{G}, a} z\right.$ $-i \omega t$ ); also see Eq. (A11) in Appendix A.

The map of different harmonics [Eq. (4)] for normal incidence, $k_{x}=k_{y}=0$, calculated with $d=680 \mathrm{~nm}$, is shown in Fig. 2, together with vacuum and substrate light cones cross sections at $\omega=1200$ and $2400 \mathrm{meV}$. As can be seen in Fig. 2, the incoming light at normal incidence with a frequency around $1200 \mathrm{meV}$ can be only transmitted or reflected. The $\left(g_{x}, g_{y}\right)=( \pm 1,0),(0, \pm 1)$ diffraction orders into the substrate soon open; see Table I. At $2400 \mathrm{meV}$ the $( \pm 1,0)$, $(0, \pm 1)$ diffractional reflexes are open into the vacuum and substrate, and the $( \pm 1, \pm 1)$ reflexes are open into the substrate. Also, the opening of $( \pm 2,0),(0, \pm 2)$ reflexes into substrate is approached.

As in case of gratings, the opening of new diffractional channels can cause peculiarities in the transmission and reflection spectra, so-called diffractive Wood's anomalies. ${ }^{13,8}$
TABLE I. Characteristic energies for the model structure (normal light incidence).

\begin{tabular}{lc}
\hline \hline Diffraction thresholds & Energy $(\mathrm{meV})$ \\
\hline$( \pm 1,0),(0, \pm 1)$, substrate & 1248.8 \\
$( \pm 1, \pm 1)$, substrate & 1766.1 \\
$( \pm 1,0),(0, \pm 1)$, vacuum & 1823.3 \\
$( \pm 2,0),(0, \pm 2)$, substrate & 2497.7 \\
$( \pm 1, \pm 1)$, vacuum & 2578.5 \\
$( \pm 2, \pm 1),( \pm 1, \pm 2)$, substrate & 2792.5 \\
& \\
Bragg resonances of the guided modes ${ }^{\text {a }}$ & \\
\hline 1st TE & 1248.1 \\
2nd TE & 1729.9 \\
3rd TE & 2359.5 \\
3rd TM & 2469.8 \\
4th TE & 2604.1 \\
4th TM & 2732.1 \\
\hline \hline
\end{tabular}

${ }^{\mathrm{a} C a l c u l a t e d}$ in empty lattice approximation.

However, in the case of a PCS with an averaged dielectric constant $\bar{\varepsilon}$ exceeding that of vacuum and substrate $\bar{\varepsilon}$ $>\left(\varepsilon_{\mathrm{s}}, 1\right)$, or covered by a guiding layer, even more pronounced features in transmission and reflection appear due to quasiguided eigenmodes in the PCS. The origin of these quasiguided modes can be easily understood within an empty lattice approximation.

\section{B. Empty lattice approximation and guided vs quasiguided eigenmodes}

In the empty lattice approximation the periodic photonic crystal slab is replaced by an effective homogeneous layer with a dielectric constant equal to the averaged dielectric susceptibility of the PCS, $\bar{\varepsilon}$; then the resulting dispersion curves are folded into the first Brillouin zone (BZ) of the original periodic structure. For a square 2D lattice we have

$$
\bar{\varepsilon}=\frac{\eta^{2} \varepsilon_{\infty}+(1+2 \eta) \varepsilon_{\mathrm{s}}}{(\eta+1)^{2}}
$$

which is near $\bar{\varepsilon} \sim 3.3$ for the model structure.

Without periodic corrugation, the guided modes in a planar waveguide are bound modes, in the sense that they are confined in the slab and decay exponentially into the vacuum and substrate outside the slab. Correspondingly, in the case of transparent materials their eigenfrequencies $\Omega\left(k_{x}, k_{y}\right)$ are real functions of the in-plane photon wave vector $\left(k_{x}, k_{y}\right)$. It is well known ${ }^{28}$ that in a planar guiding layer on a substrate, there are $j=1,2, \ldots$ transverse electric (TE) and higherfrequency transverse magnetic (TM) waveguide modes. Electric field in TE modes and magnetic field in TM modes are parallel to the slab plane, and the modes exist at frequencies above the corresponding frequency cutoffs, 


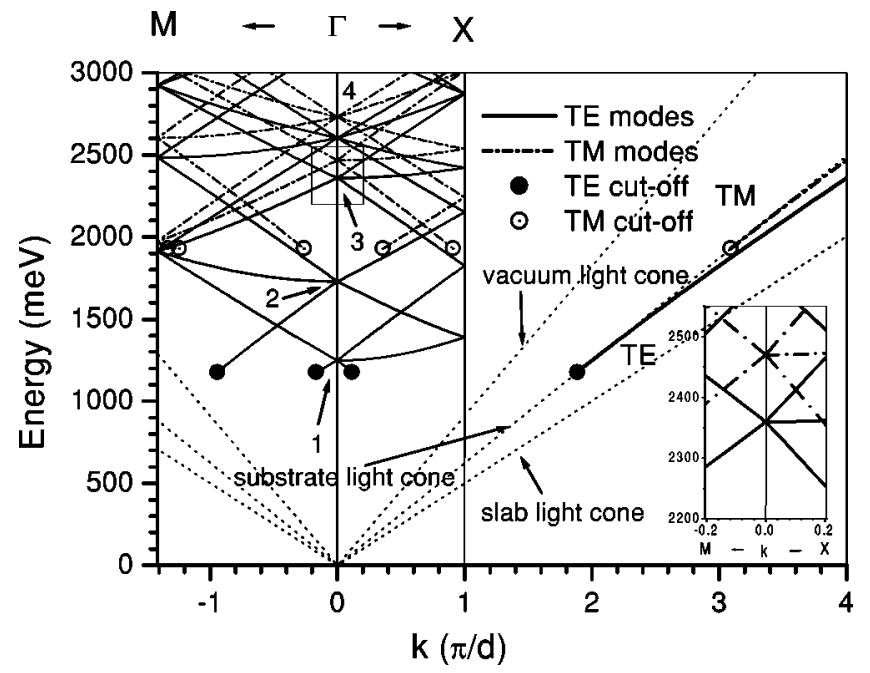

FIG. 3. The lowest TE and TM guided modes in an effective homogeneous waveguide with an averaged dielectric constant (right); same modes folded into the first BZ of $d=680 \mathrm{~nm}$ square lattice (left). The dispersion of folded branches is shown along $\Gamma-X$ and $\Gamma-M$ directions. Four lowest TE (1-4) and two lowest TM (3 and 4) Bragg resonances in the $\Gamma$ point are shown. Inset: the magnified region around the third Bragg resonance.

$$
\Omega_{j}^{\text {(cutoff })}=\frac{c}{L_{z} \sqrt{\bar{\varepsilon}-\varepsilon_{\mathrm{s}}}}\left(\arctan \epsilon \sqrt{\frac{\varepsilon_{\mathrm{s}}-1}{\bar{\varepsilon}-\varepsilon_{\mathrm{s}}}}+j \pi\right),
$$

where $\bar{\varepsilon}$ and $\varepsilon_{\mathrm{s}}$ are the dielectric constants of the guided layer and the substrate, respectively, and $\epsilon=1$ for TE modes and $\bar{\varepsilon}$ for TM modes. The energy dispersion of TE and TM modes is found from transcendent equations, which we give here for self-consistency:

$$
\tan \left(\bar{\beta} L_{z}\right)=\frac{\bar{\beta}\left(\beta_{\mathrm{s}}+\beta\right)}{\bar{\beta}^{2}-\beta_{\mathrm{s}} \beta}
$$

for TE modes and

$$
\tan \left(\bar{\beta} L_{z}\right)=\frac{\bar{\varepsilon} \bar{\beta}\left(\beta_{\mathrm{s}}+\varepsilon_{\mathrm{s}} \beta\right)}{\varepsilon_{\mathrm{s}} \bar{\beta}^{2}-\bar{\varepsilon}^{2} \beta_{\mathrm{s}} \beta}
$$

for TM modes, where $\bar{\beta}=\sqrt{\bar{\varepsilon} k_{0}^{2}-k^{2}}, \quad \beta_{\mathrm{s}}=\sqrt{k^{2}-\varepsilon_{\mathrm{s}} k_{0}^{2}}, \quad \beta$ $=\sqrt{k^{2}-k_{0}^{2}}, k_{0}=\Omega / c$, and $k=\sqrt{k_{x}^{2}+k_{y}^{2}}$.

The lowest $j=1 \mathrm{TE}$ and TM waveguide modes and their frequency cutoffs in an effective planar waveguide, calculated from Eqs. (9) and (10) and Eq. (8), respectively, with $L_{z}=120 \mathrm{~nm}$ and $\bar{\varepsilon}$ from Eq. (7), are shown in Fig. 3 (righthand side).

The guided modes in the effective planar waveguide are completely confined in a slab, and formally have an infinite lifetime. The introduction of corrugation can couple the guided modes with photon continua in vacuum and substrate. As a result, the guided modes acquire a finite lifetime inside the light cones in the first Brillouin zone and become quasiguided (or leaky) modes. ${ }^{5}$ Due to the $1 \mathrm{D}$ or $2 \mathrm{D}$ periodicity of the corrugated waveguide, the frequency dispersion diagram of the quasiguided modes consists of allowed bands separated by photonic stop bands in the center and borders of the first BZ.

The origin of the allowed bands can be understood in the empty lattice approximation by means of folding the guided modes of the effective planar waveguide (Fig. 3, right hand side) into the first BZ of the PCS. The resulting empty lattice bands for the model system are shown in Fig. 3, left-hand side.

It is seen in Fig. 3 that four families of bands are formed in this energy region near the center of the first BZ (the first to the fourth resonances in the $\Gamma$ point hereafter, marked in Fig. 3 by the corresponding numbers); also see the energies of the bands in the $\Gamma$ point in Table I. First of all, let us note all these bands lie well above the vacuum and substrate light cones in the first BZ due to relatively high TE and TM cutoff energies in the model structure. Thus in the model structure there is no purely guided eigenmode of the type analyzed, e.g., in Ref. 5. Also note that, due to a large difference between TE and TM cutoffs, only TE-type modes participate in the two lower resonances.

The lowest band in the $\Gamma$ point is the standing wave originating from the Bragg resonance of the lowest TE branch of the effective waveguide at $\mathbf{g}=( \pm 1,0),(0, \pm 1)$ points in the reciprocal lattice. In the empty lattice approximation it is a degenerate quartet. Along the $\Gamma-X$ direction this band splits off into two fast modes [originating from $(1,0)$ and $(-1,0)$ ] and a doubly degenerate slow mode [from $(0, \pm 1)]$. Along the diagonal direction $\Gamma-M$ both bands are degenerate doublets.

The second band corresponds to the $( \pm 1, \pm 1)$ TE resonance; for this resonance the behavior along $\Gamma-X$ and $\Gamma-M$ is inverted in comparison with the first resonance case. The next bands lie well above both TE and TM cutoffs, so the next resonances occur both in TE and TM modes. The third band, corresponding to $\mathbf{g}=( \pm 2,0),(0, \pm 2)$, is a group of TE and TM modes shown in the rectangle (also see the magnified inset in Fig. 3), and the fourth band has $\mathbf{g}=( \pm 2$, $\pm 1),( \pm 1, \pm 2)$.

The symmetry of bands near the third and first resonances is the same. We will see below that the third band causes the strongest features in the transmissivity spectra of the model structure. Thus in this paper we will focus mostly on this resonance. Its behavior is somewhat typical at a square PCS. For the subsequent discussion it is very instructive to consider the properties of slow and fast modes, e.g., along the $\Gamma-X$ direction. The slow modes along $\Gamma-X$, being produced by transverse waves with wave vectors $\left(k_{x}, k_{y}\right)=(0, \pm 2)$ and thus propagating along $\hat{\mathbf{y}}$, have an electric (magnetic) field polarized predominantly along $\hat{\mathbf{x}}$ for TE- (TM)-type resonance. The fast modes are predominantly polarized along $\hat{\mathbf{y}}$.

Depending on the parameters chosen, the energy positions of the bands are changed. But the properties do not change qualitatively from the situation analyzed here with this set of parameters. Similarly there are resonances at the boundaries of the first BZ, and also band crossings inside the BZ. 
The periodic corrugation of the layer makes two important changes with this folded picture. First of all, the degeneracy of the crossing modes in resonances is partially removed, due to the differences in the electromagnetic field distributions in modes of different types, depending on the symmetry properties of the periodic lattice. As a result, stop bands appear in the center, at the boundaries of BZ, and at some crossing points. This is typical of any periodic structure, including photonic crystals. Second, most of the eigenmodes discussed above become radiative or leaky, due to the opening of the interaction between them and photon continua in vacuum and substrate, which is a very important difference from the case of bulk photonic crystals. In order to distinguish the leaky modes of waveguide origin from all other types of leaky modes in the PCS (e.g., Fabry-Perot modes), we call them "quasiguided modes." As we will see, such quasiguided modes are typically characterized by relatively long lifetimes and may have large quality factors. Thus the field in such modes becomes resonantly enhanced inside the PCS. This opens many interesting possibilities to use this resonantly increased field for, e.g., resonant amplification of nonlinear optical effects or controlling the radiative lifetimes of optical transitions in the PCS.

On the other hand, the coupling between photon continua and quasiguided modes allows one to excite these modes resonantly (in energy and angle of incidence) via external light. The resonant excitation of the quasiguided modes manifests itself in appearance of well-pronounced dips in the transmission spectra. The change of energy position of dips with change of incidence angle $\vartheta$ reflects the dispersion of the quasi-guided modes as will be discussed in Sec. IV A. In the experiments, the measurement of the transmission spectra at different angles brings an information on the dispersion of quasiguided modes. ${ }^{20,6}$ By utilizing the advantage of such configuration, e.g., in Refs. 20 and 18, a strong coupling between an exciton resonance and 1D and 2D corrugated quasiguided modes has been experimentally demonstrated at room temperature in structures, fabricated via imbedding into a periodically patterned quartz substrate of inorganicorganic semiconductor with a very strong polariton coupling.

As already mentioned, the model structure does not support purely guided modes below the light cones in the first $\mathrm{BZ}$ at all. However, as will be shown later, in this particular system a pair of nonleaky modes are still allowed in the $\Gamma$ point, originating from the first resonance in Fig. 3; see Sec. IV A. Because these modes appear well inside the light cones, their nonleaky character is entirely connected with their symmetry properties. Such a situation was analyzed previously in Ref. 9. However, for higher resonances all the modes are leaky, including those optically inactive in normal direction, because the interaction with higher Bragg harmonics becomes open for the latter.

\section{SCATTERING-MATRIX THEORETICAL DESCRIPTION OF THE OPTICAL PROPERTIES OF PCS'S}

\section{A. Formulation of the method}

In order to formulate the scattering matrix formalism for the PCS, we have to describe the input and output states of the scattering problem, and to define the scattering matrix which transfers the input state into the output one. In the exact decomposition the number of Bragg harmonics needed for the full description of the electromagnetic field in the system is infinite, but in numerical calculations we take a finite number of harmonics. Specifically, for a square 2D lattice we take $N_{g}=\left(2 g_{\max }+1\right)^{2}$ harmonics with $\left|g_{x, y}\right|$ $\leqslant g_{\max }$. To describe an electromagnetic field of each harmonic we need four independent scalar amplitudes (for example, $E_{x}, E_{y}, H_{x}$, and $H_{y}$ ), so the total field is fully specified via $4 N_{g}$ scalar amplitudes of Bragg harmonics, $\mathcal{A}_{\mathbf{G}}^{ \pm}$, where the upper sign stands for the sign in Eq. (3), see the introduction of amplitudes in Appendix B.

For propagating harmonics, $k_{\mathbf{G}, \mathbf{v}}^{+}$waves in vacuum semispace and $k_{\mathbf{G}, \mathrm{s}}^{-}$in the substrate should be considered as an incoming plane waves. Indeed, both types of harmonics propagate toward the PCS layer; see Fig. 1. The exponential plane waves do not propagate, so we have to use another criterium in order to deal with them. Note that the $k_{\mathbf{G}, \mathbf{v}}^{+}$and $k_{\mathbf{G}, \mathrm{s}}^{-}$exponential harmonics are increscent solutions when $z$ $\rightarrow-\infty$ and $z \rightarrow \infty$, respectively. For a physical scattering problem we have to put the amplitudes of such increscent plane waves zero at $z=-\infty$ and $\infty$, respectively. It is important that including the amplitudes of increscent waves into the incoming vector also matches the general motivation to use the scattering matrix approach. Then, as discussed, e.g., in Ref. 23 (also see in Appendix D), the components of the $S$ matrix do not contain exponentially large components, and the $S$-matrix calculation scheme becomes numerically convergent.

From this consideration it follows that, for the scattering problem, it is convenient to define the incoming and outgoing states via $4 N_{g}$-dimensional vectors $B_{\text {in }}$ and $B_{\text {out }}$, composed of the amplitudes of the incoming and outgoing plane waves:

$$
\vec{B}_{\text {in }}=\left(\begin{array}{c}
\overrightarrow{\mathcal{A}}_{\mathrm{v}}^{+} \\
\overrightarrow{\mathcal{A}}_{\mathrm{s}}^{-}
\end{array}\right), \quad \overrightarrow{\mathrm{B}}_{\text {out }}=\left(\begin{array}{c}
\overrightarrow{\mathcal{A}}_{\mathrm{s}}^{+} \\
\overrightarrow{\mathcal{A}}_{\mathrm{v}}^{-}
\end{array}\right) .
$$

In this representation, the only nonzero element of incoming amplitude column $\vec{B}_{\text {in }}$ is the amplitude of the incident wave, $\left(\vec{B}_{\text {in }}\right)_{\mathbf{G}=0}$, while the outgoing column $\vec{B}_{\text {out }}$ contains amplitudes of harmonics, proportional to $\exp \left(-i k_{z, \mathbf{G}, \mathrm{v}} z-i \omega t\right)$ at $z<0$ (reflected and evanescent waves) and to $\exp \left(i k_{z, \mathbf{G}, \mathrm{s}} z\right.$ $-i \omega t)$ at $z>L_{z}$ (transmitted and evanescent waves).

The full description of the optical response of the system at frequency $\omega$ can be done by calculating the full scattering matrix of the system $\mathrm{S}$, which connects the incoming vector of amplitudes with the outgoing one:

$$
\overrightarrow{\mathrm{B}}_{\text {out }}=\mathrm{SB}_{\text {in }} \text {. }
$$

Thus, the full $S$ matrix is a $\left(4 N_{g} \times 4 N_{g}\right)$-dimensional matrix in our case.

In this paper we deal with $N_{g^{-}}, \quad 2 N_{g^{-}}$, and $4 N_{g}$-dimensional hypervectors and $\left(N_{g} \times N_{g}\right)^{-}, \quad\left(2 N_{g}\right.$ $\left.\times 2 N_{g}\right)$-, and $\left(4 N_{g} \times 4 N_{g}\right)$-dimensional matrices. In order to distinguish vectors and matrices of different dimensionality, 
hereafter we denote such hypervectors and matrices by italic, calligraphic, and open face letters, respectively, with arrows to denote the vectors. For example, in Eq. (11) the total amplitude $\overrightarrow{\mathrm{B}}$ is a $4 N_{g}$-dimensional vector, whereas the partial amplitudes $\overrightarrow{\mathcal{A}}^{ \pm}$are $2 N_{g}$-dimensional vectors. In order to calculate the optical properties of PCS, we do the following

(1) Split the system into the layers, homogeneous along the $z$ axis ( 3 layers in case of the model structure shown in Fig. 1), and solve Maxwell's equations in each layer via decomposition into partial plane waves (see the full description of the procedure in Appendix A).

(2) For each layer, construct material matrices (Appendix B) to calculate the in-plane components of the local electromagnetic fields via the amplitudes of partial waves. Propagation and interface transfer matrices to be constructed as well, to connect amplitudes in different parts of the structure;

(3) Construct the total scattering matrix $\mathrm{S}$ of the whole structure, connecting the incoming and outgoing vector amplitudes via the iterative procedure by Ko and Inkson, ${ }^{23}$

(4) Calculate, via the components of $S$ matrix, the optical properties of the system such as transmission, reflection, absorption, and the corresponding spatial distributions of the electromagnetic fields.

For the sake of consistency, we give the full formulation of transition from the $T$-matrix to the $S$-matrix formalism in Appendix D. In Appendix C we specify the input amplitudes for the problem of light transmission, reflection, and deflection. We also give the analytical form of all generic matrices for spatially homogeneous slab in Appendix F.

Knowledge of the scattering matrix $\mathrm{S}$ of the whole system and of the input amplitude vector $\vec{B}_{\text {in }}$ allows us to calculate the reflection, transmission, and absorption coefficients. The simplest way to do this is to calculate the $z$-components of the Poynting vectors for incoming, reflected, transmitted and all deflected waves, as shown in Appendix E,

$$
R=-\frac{P^{(\mathrm{r})}}{P^{(\mathrm{in})}}, \quad T=\frac{P^{(\mathrm{t})}}{P^{(\mathrm{in})}}, \quad D=\frac{P^{(\mathrm{d}, \mathrm{s})}-P^{(\mathrm{d}, \mathrm{v})}}{P^{(\mathrm{in})}},
$$

where the expressions for the different Poynting vector components; see Eq. (E1). Note that the Poynting vector components of the waves reflected and deflected into vacuum enter Eqs. (13) with negative sign (because they counter-propagate against the incoming wave).

\section{B. A numerical example: transmissivity of the model structure}

Let us turn now to the analysis of the transmissivity of the model structure, calculated via the scattering matrix method introduced in Sec. III. The calculated transmission spectrum of the model structure for normal incidence and polarization along $\hat{\mathbf{y}}$ is shown in Fig. 4.

Let us note here that our numerical algorithm converges very fast (at least for dielectric materials and for a calculation of the transmissivity). Actually, there are three curves shown in Fig. 4, for $g_{\max }=3,5$, and 6, but the difference is smaller than the lines thickness; see the inset in Fig. 4. The number of harmonics taken are 49,121 , and 169 , respectively. Thus our electric field based scheme becomes more rapidly con-

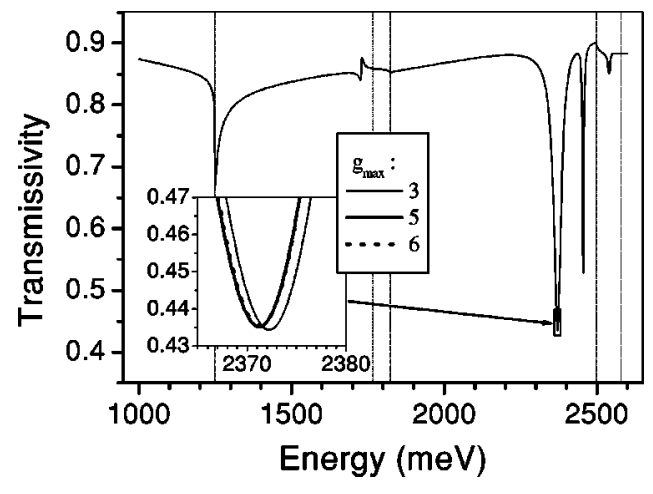

FIG. 4. The calculated transmissivity of the model structure at normal incidence. Vertical dashed lines mark the energies of diffraction thresholds; see Table I. In order to illustrate the fast convergency of the method developed, three spectra are actually shown in this figure, calculated with $g_{\max }=3,5$, and 6 , but the difference between them is less than the line thickness; see the magnified part of the main dip in the inset.

verging than the magnetic field based described in Ref. 7: 49 harmonics give a suitable approximation for transmissivity with an accuracy in calculating the dips energies within 1 $\mathrm{meV}$. Note that within our method we do not need any additional orthogonalization procedure, ${ }^{7}$ and employ the $\varepsilon_{\mathbf{G G}^{\prime}}$ matrix directly (also see the discussion in Appendix A). During the preparation of this paper, as well as during working on the previous publications ${ }^{17,18}$ we have not found any example of nonconvergency of this method, at least for dielectric structures, including the cases with accounting for the polariton pole in the dielectric susceptibility.

As seen in Fig. 4, the calculated transmissivity shows three groups of features around 1200, 1700, and 2350-2700 $\mathrm{meV}$. This is in close correspondence with the discussion of the diffraction orders thresholds (vertical dashed lines in Fig. 4) and quasiguided bands given in Sec. II (also compare with Table I). The lowest-energy cusplike feature is seen exactly at the energy of $( \pm 1,0),(0, \pm 1)$ diffraction threshold into substrate, and the shape of this feature is typical for a diffractive Wood anomaly. There is no additional feature seen here which may be attributed to the excitation of the first TE-type quasiguided band, at least at normal incidence. (As the further analysis shows, this is due to the proximity of this Bragg resonance to the TE cutoff energy. Still, we do not dwell into complicated details of the quasiguided mode cutoffs in this paper.)

The next feature in Fig. 4, which appears around 1700 $\mathrm{meV}$, consists of a hardly seen cusp at the threshold of $( \pm 1, \pm 1)$ diffraction into substrate, a small but visible cusp at the threshold of $(0, \pm 1),(0, \pm 1)$ diffraction into vacuum, and a feature at lower energy, which can be attributed to the interaction with the second TE Bragg quasiguided band. The most prominent features, consisting of two strong dips around $2400 \mathrm{meV}$, as we show below, are due to the interaction of the incoming light with the third Bragg quasiguided band. An inverted cusp corresponding to the $( \pm 2,0),(0$, \pm 2 ) diffraction threshold into the substrate is seen above them. A smaller rightmost dip is due to the interaction with 


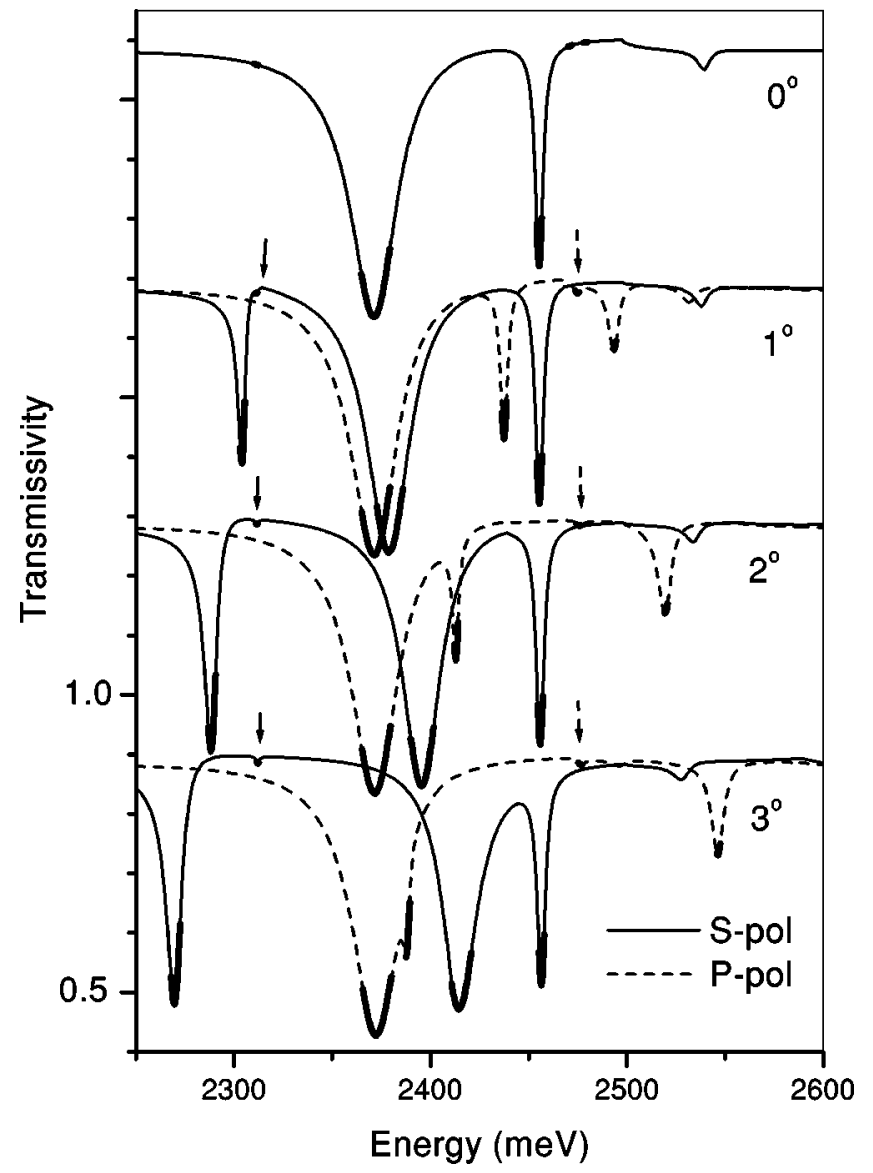

FIG. 5. The calculated transmissivity of the model structure near the third Bragg resonance in $S$ and $P$ polarizations for different angles of incidence. The parts of the transmission curves between $\Omega-\gamma / 2$ and $\Omega+\gamma / 2$ are boldfaced, in order to emphasize the relation between the position and width of the dips and the eigenmode frequency and linewidth. Here $\Omega$ and $\gamma$ are the real and imaginary parts of the corresponding quasi-guided mode eigenfrequency $\Omega$ $-i \gamma$; see Sec. IV B.

the fourth Bragg quasiguided band, and a very small cusp corresponding to the $( \pm 1, \pm 1)$ diffraction threshold in vacuum can be hardly seen above it. In what follows, we focus mainly on the behavior around $2400 \mathrm{meV}$, where the main features appear to be connected with the TE and TM quasiguided modes of the third Bragg band.

Under the oblique incidence the transmission spectra become even more complicated: new dips appear, and some of the dips shift to red, whereas the other shift to blue. The complicated behavior of the dips around $2400 \mathrm{meV}$ is shown in for angles of incidence $\vartheta=0-3^{\circ}$ and $\varphi=0$ in Fig. 5 for $S$ and $P$ polarizations. Several important trends in the dips behavior can be seen in this Fig. 5:

(1) At $\vartheta=0$, which corresponds to $\Gamma$ point, we see two dips in the transmissivity: the main dip at lower energy and a smaller and narrower one at higher energy; due to the square symmetry, the transmission spectra in $S$ and $P$ polarizations coincide.

(2) In $S$ polarization, with the increase of $\vartheta$ we see that the main dip rapidly shifts to higher energies, while the smaller dip appears to stay unshifted at small angles. At the same time, a couple of new dips arise at the lower-energy side of the main dip: another well-pronounced dip which rapidly shifts to lower energies, and a small dip which appears not to shift with the angle of incidence. (This dip is marked by vertical solid arrows in Fig. 5.)

(3) In $P$ polarization, with the increase of $\vartheta$ the main dip does not shift; the smaller dip shifts rapidly to lower energies; two new dips arise at the higher energy side of the smaller dip: a new well-pronounced dip, which shifts rapidly to higher energies, and a small dip which appears not to shift with the angle of incidence. (This dip is marked by vertical dashed arrows in Fig. 5.)

This behavior can be quantitatively understood in the model of excitation of different quasi-guided modes in the PCS.

\section{QUASIGUIDED MODES}

It appears that the most prominent features in transmission of light through a PCS are connected with the properties of quasiguided (or leaky) modes which can propagate along the PCS. Unlike the guided modes in a homogeneous waveguide, they have finite radiative linewidth due to the coupling with vacuum and substrate photonic continua. In Sec. II we showed the physical origin of such eigenmodes using the empty lattice approximation. Now we can calculate their properties such as dispersion of eigenenergy, optical activity, and electromagnetic field distribution.

\section{A. Quasiguided modes and the scattering matrix}

In this subsection we apply the scattering matrix formalism to calculate the frequency and linewidth dispersion of quasiwaveguide modes as well as the distribution of the electromagnetic field in such modes. The eigenmodes of the system are the nontrivial solutions of Eq. (12) under the condition of zero input amplitudes $\vec{B}_{\text {in }}=0$. Thus the amplitudes of eigenmodes can be found from the solution of the homogeneous linear problem

$$
\mathrm{S}^{-1} \overrightarrow{\mathrm{B}}_{\text {out }}=0
$$

This means that

$$
\operatorname{det} \mathrm{S}^{-1}\left(\omega, k_{x}, k_{y}\right)=0
$$

is the main equation for calculating the eigenfrequences.

In a uniform slab, which is a planar waveguide, the guided eigenmodes have real eigenfrequences $\Omega$, and their electromagnetic field is confined inside the slab, exponentially decaying outside. Contrastingly, in the case of periodic corrugation the eigenmodes couple with photonic continuum in vacuum and substrate. Thus the eigenmodes become leaky, or quasiguided. Only the lower-energy eigenmodes lying below the substrate light cone in the first Brillouin zone retain a purely guided character. ${ }^{5}$ The electromagnetic field of quasiguided modes acquires a radiative component which diverges exponentially at $z \rightarrow \pm \infty$, and their eigenfrequencies become complex. Although such solutions seem to be not physical, it is not the case actually; see, e.g., Ref. 29. These exponentially growing (with $z \rightarrow \pm \infty$ ) solutions are physical 
because, in the time domain, the exponential growth is cancelled by a time-dependent multiplier $\exp (-|\operatorname{Im} \Omega| t)$. In other words, the time-space dependence of the electromagnetic field in such leaky modes is $\propto \exp [|\operatorname{Im} \Omega|(z-c t) / c]$ and describes the propagating front of the decaying state. Thus, in order to calculate the properties of the quasi-guided modes, we should define the $S$ matrix in the complex frequency plane.

From the causality properties, the $S$ matrix is the analytical function in the upper frequency half-plane, and it may have poles in the lower half-plane at $\Omega-i \gamma, \gamma \geqslant 0$, including a real axis in case of transparent (nonabsorptive) materials. Such poles correspond to physical eigenmodes with an electromagnetic field $\propto \exp (-i \Omega t-\gamma t)$ decaying with $t \rightarrow \infty$, and $\gamma$ is their inverse lifetime. Among all possible solutions, only those with $\gamma \ll \Omega$ are of the physical interest.

The $S$ matrix for each given wave vector $\left(k_{x}, k_{y}\right)$ [Eq. (1)] can be directly constructed on the complex plane via the analytical continuation from the real axis of energies, where it is defined unambiguously (see Appendix D). Thus the only thing which is left is to find the roots of Eq. (15), $\Omega\left(k_{x}, k_{y}\right)-i \gamma\left(k_{x}, k_{y}\right)$, which are close to the real energy axis.

Here it is important to note that the $S$ matrix, defined on the real axis (Appendix D) allows several different analytical continuations from different segments of real $\omega$ separated by diffraction thresholds. Let us discuss this point in more details.

As we discussed in Sec. III A, the $k_{\mathbf{G}, \mathrm{v}}^{+}$and $k_{\mathbf{G}, \mathrm{S}}^{-}$harmonics are the solutions, either propagating toward the PCS or exponentially increscent outside the PCS for any $\mathbf{G}$ on the real $\omega$ axis. Thus they comprise the list of the incoming waves.

On the complex $\omega$ plane, and for "propagating" harmonics, i.e., for $\operatorname{Re} k_{z, \mathbf{G}, a}^{2}>0$, the conventional definition ${ }^{27}$ of the complex square root in Eq. (5) contains a cut below $\operatorname{Re} k_{z, \mathbf{G}, a}^{2}<0$, and provides an analytical behavior of $k_{z, \mathbf{G}, a}$ when $k_{z, \mathbf{G}, a}^{2}$ crosses the real positive axis. However to ensure the analytical continuation of $k_{z, \mathbf{G}, a}$ (and, consequently, of the $S$ matrix) when the harmonics become "exponential," $k_{z, \mathbf{G}, a}^{2}<0$, we have to redefine a cut in the square-root function: we move it from the quadrant below the negative real axis on $k_{z, \mathbf{G}, a}^{2}$ complex plain, as in the conventional definition, to, e.g., the quadrant below the positive real axis.

The question of whether $k_{z, \mathbf{G}, a}^{2}$ crosses the positive or negative real axis is equivalent to whether the $\mathbf{G}$ diffraction channel is open at the given energy or not. Thus we can conclude that the set of definitions of the square roots depends on the harmonics energy and is fixed for a given segment, bounded by neighboring diffraction thresholds.

If the analytical continuation of the $S$ matrix (built from some segment of real $\omega$ in accordance with the above-stated rules) contains some poles below this segment, and all the poles lie far from the ends of the segment (i.e., diffraction thresholds), then the single particular sheet of the $S$ matrix is sufficient for a complete understanding of the PCS optical properties in this energy region. In the model structure, such a situation takes place, e.g., for the third Bragg resonance.

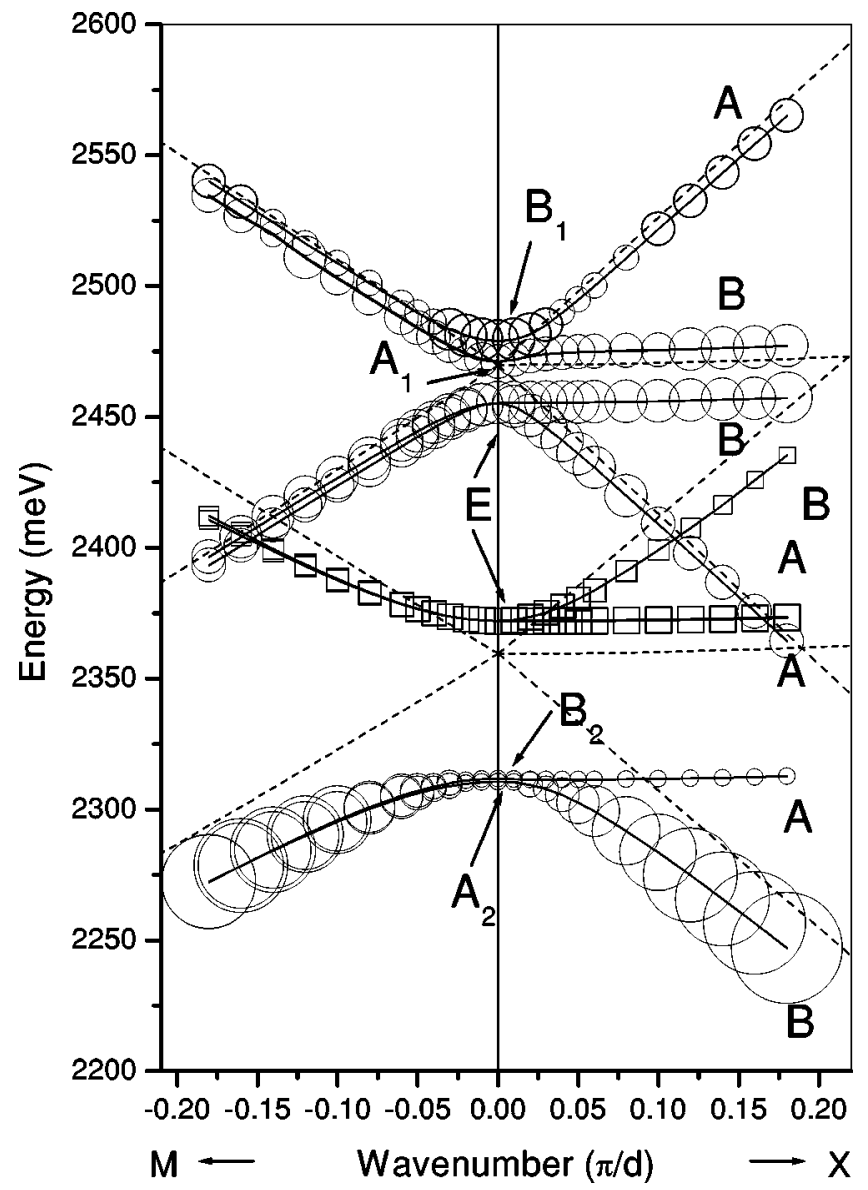

FIG. 6. The calculated dispersion of the TE-TM quasiguided modes octet along $\Gamma-X$ and $\Gamma-M$ directions for the third resonance near the $\Gamma$ point. The labels $E, A_{1,2}$ and $B_{1,2}$ (the irreducible representations of the point group $C_{4 v}$ ) and $A$ and $B$ (irreducible representations of the point group $C_{1 h}$ ) show the symmetry of the electric field of the corresponding eigenmode. The dashed lines show the quasiguided modes in empty lattice approximation; also see the insert in Fig 3. The size of squares is equal to the imaginary part of the corresponding eigenenergy, while the diameter of the open circles is ten times the corresponding imaginary part.

However, near the cutoffs of the quasiguided eigenmodes the corresponding poles approach the diffraction thresholds, and more than one sheet of the $S$ matrix become physically meaningful. As far as we know, an example of such a complicated behavior was analyzed for the first time in Ref. 30 . In the model structure such a situation takes place, e.g., near the first Bragg resonance. However, in this paper we do not analyze this situation and focus mainly on a simpler situation around the third Bragg resonance, when only one physical sheet of the $S$ matrix is sufficient to describe the physics.

\section{B. A numerical example: quasiguided modes in the model structure}

In Fig. 6 we show the dispersion curves of the TE-TM octet calculated via Eq. (15) for the model structure near the $\Gamma$ point along $\Gamma-X$ and $\Gamma-M$ directions. Our numerical analysis shows that for this particular system the third reso- 
TABLE II. The energies of the third Bragg eigenmodes in the $\Gamma$ point.

\begin{tabular}{lcc}
\hline \hline Type & $\Omega(\mathrm{meV})$ & $\gamma(\mathrm{meV})$ \\
\hline Lower TE singlet & 2310.7 & 1.0 \\
Higher TE singlet & 2311.8 & 1.0 \\
TE doublet & 2372.0 & 14.5 \\
TM doublet & 2455.4 & 2.4 \\
Lower TM singlet & 2471.1 & 1.9 \\
Higher TM singlet & 2478.9 & 2.2 \\
\hline \hline
\end{tabular}

nance in $\Gamma$ consists of two quartets: a lower TE-like quartet, composed of two singlets (for the electric field, $A_{2}$ and $B_{2}$ irreducible representations of point group $C_{4 v}$ ) and one doublet $(E)$, and a higher TM-like quartet, composed of one doublet $(E)$ and a pair of singlets $\left(A_{1}\right.$ and $\left.B_{1}\right)$ at higher energy. Let us recall here that the $A_{1}$ irreducible representation of $C_{4 v}$ is invariant over all rotations and mirror reflections of $C_{4 v} ; A_{2}$ is invariant over all rotations and odd over all mirror reflections; $B_{1}$ is odd over $90^{\circ}$ rotations and $\sigma_{d}$ (diagonal) reflections; $B_{2}$ is odd under $90^{\circ}$ rotations and $\sigma_{x, y}$ reflections; and components of a doublet $E$ are connected with each other via a $90^{\circ}$ rotation and $\sigma_{d}$ reflections; see the classification of the irreducible representations for different points of the first Brillouin zone of the square 2D lattice in, e.g., Ref. 31. The calculated real and imaginary parts of corresponding eigenenergies in the $\Gamma$ point are given in Table II.

The spatial distributions of the electric and magnetic fields in TE singlets on the vacuum-side surface of the model structure are shown in Figs. 7 and 8. Note that here we give the top vertical view, so only the $(x, y)$ components of the fields are seen. The same data are shown in Fig. 9 for the $x$-polarized component of TE doublet. The electric and magnetic fields are shown in Figs. 7-9 at moments of time $t_{E}$ and $t_{H}$, respectively, when the intensity of the corresponding field is at a maximum. It appears that these moments are separated by one-fourth of the period of the corresponding eigenmode, $t_{E}-t_{H} \approx T / 4, T=2 \pi / \Omega$. Thus all the modes are mostly standing electromagnetic waves.

The type of each irreducible representation is obvious from this figure. Let us note that the one-dimensional irreducible representations of the eigenmode vector field $\mathbf{E}$ and the pseudovector field $\mathbf{H}$ have different characters of mirror reflections in $C_{4 v}$.

The size of the circles in Fig. 6 indicates the imaginary part of the corresponding eigenenergy (in case of open circles it is magnified ten times magnified); also see the last column in Table II. The $C_{4 v}$ symmetry properties imply that all the singlet states are optically inactive in the $\Gamma$ point (in normal direction), and only the properly polarized components of the doublets are optically active. This explains why only two dips are seen at $\vartheta=0$ in Fig. 5: only one doublet component is active per each quartet.

The widths of optically active states are larger than that of inactive states. At normal incidence, the TE doublet linewidth is one order of magnitude larger than widths of inactive TE singlets and all TM states $(\sim 15$ and $1-2 \mathrm{meV}$, re-
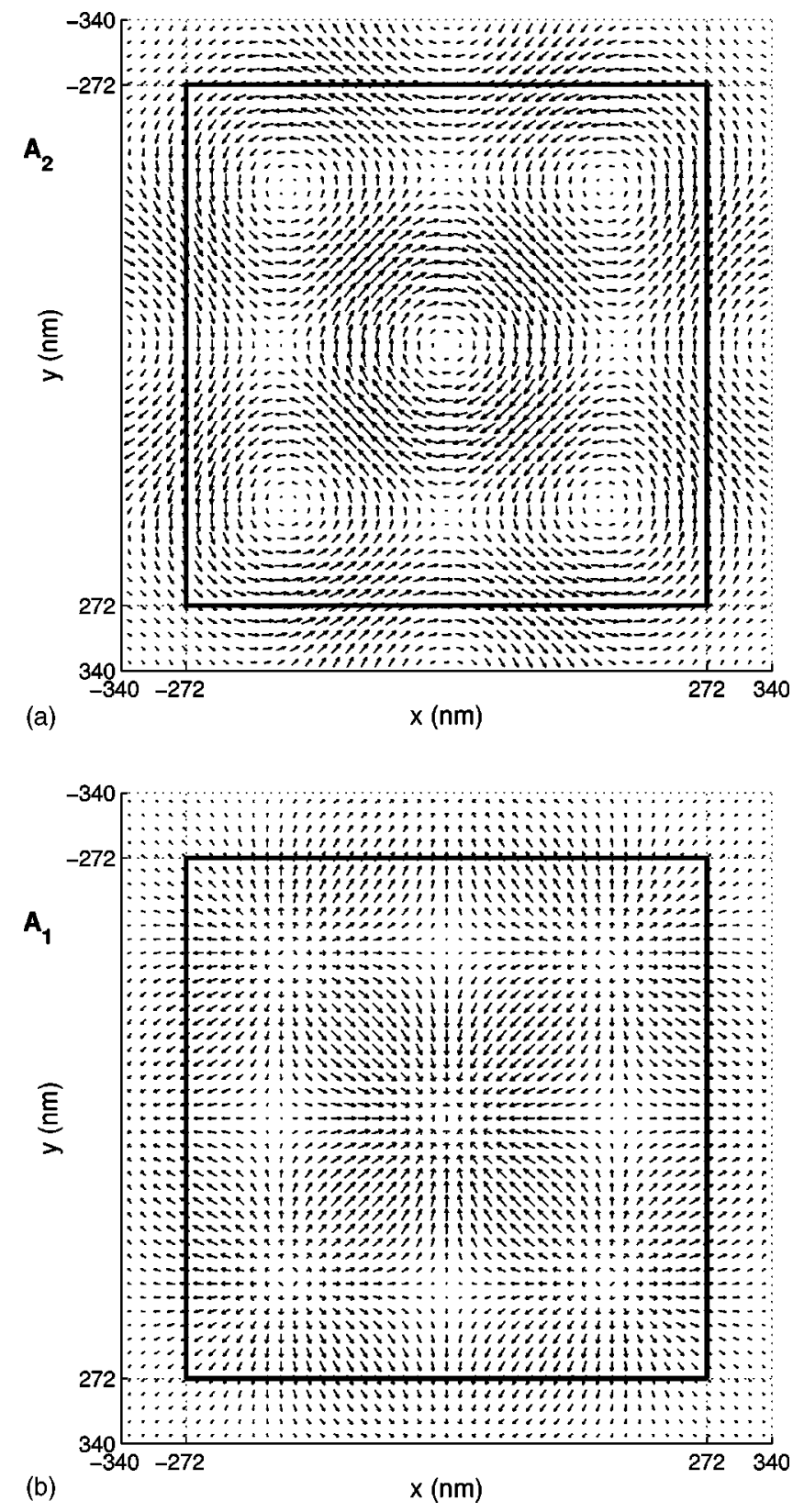

FIG. 7. Electric (a) and magnetic (b) fields on the top (vacuum side) of the PCS in the lower TE singlet $-A_{2}$ and $A_{1}$ representations, respectively. One unit cell of a $2 \mathrm{D}$ square lattice is shown. The active material is inside the thick square.

spectively; see Table II). This corresponds to the most pronounced dip in the transmission. The quality factors of the states are as large as $(1-2) \times 10^{3}$ for TM and TE singlet states, and $\sim 160$ for the TE doublet, which corresponds to the resonant increase of the field in the modes in 30-40 and $\sim 13$ times, respectively.

Note that the linewidths of optically inactive states are nonzero at normal incidence. At this point we should emphasize a very important difference concerning the properties of the inactive modes, belonging to the third resonance (shown in Fig. 6) and to the first resonance, reported previously in Ref. 9. In the case of the first resonance, only the interaction with the main harmonic is allowed; see the light cones cross- 

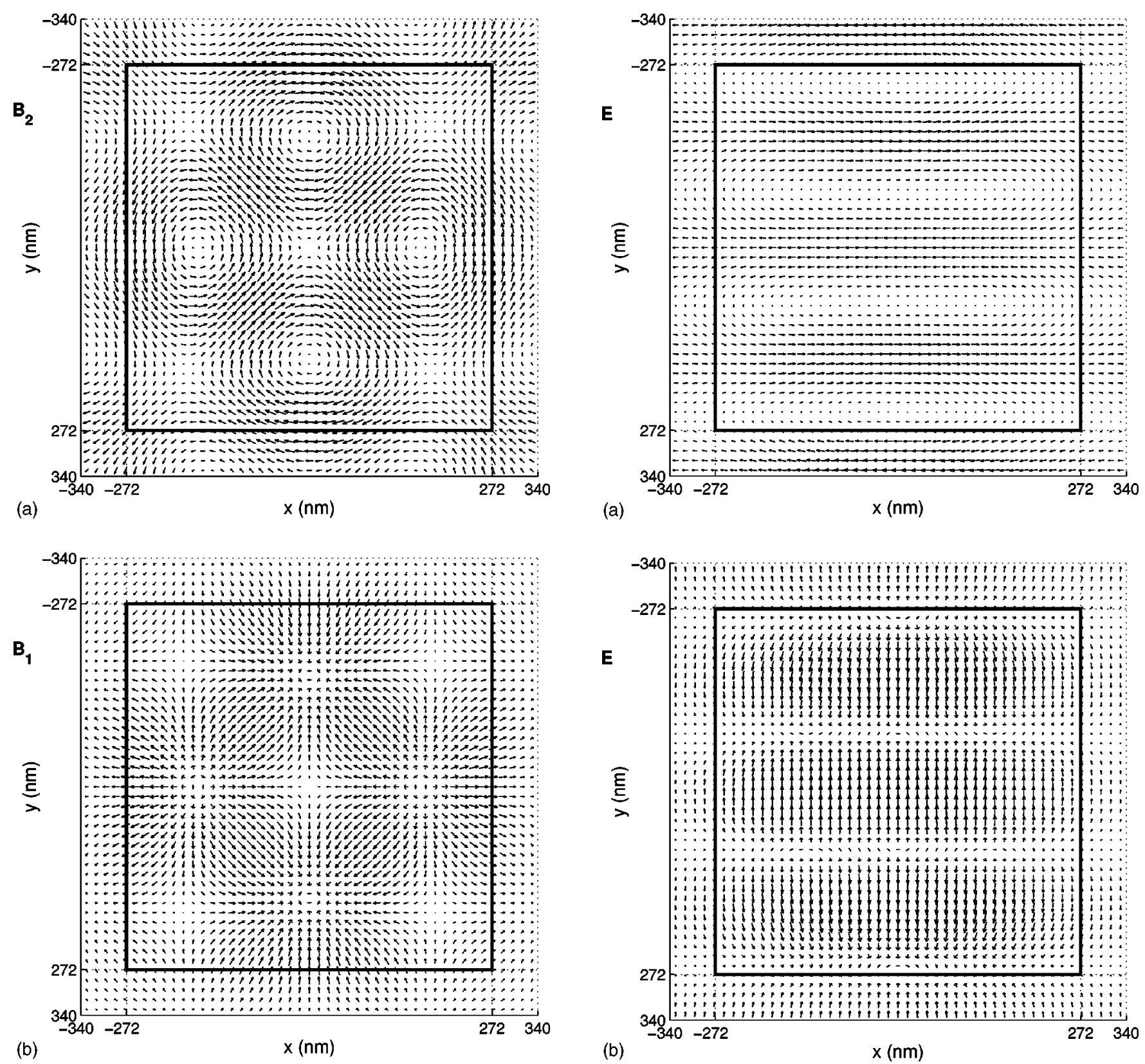

FIG. 8. Electric (a) and magnetic (b) fields in the upper TE singlet- $B_{2}$ and $B_{1}$ representations, respectively.

sections marked by the thin lines in Fig. 2. When this interaction is forbidden by the symmetry-for the singlet antisymmetric modes-such modes have zero linewidth and become nonleaky. ${ }^{9}$

Conversely, in the case of the third resonance the linewidth of inactive modes is nonzero: although such modes are optically inactive in normal direction, they can radiate $( \pm 1,0),(0, \pm 1)$ Bragg harmonics into the vacuum and substrate, and $( \pm 1, \pm 1)$ harmonics into the substrate; see the light cone cross sections marked by thick lines in Fig. 2. At $k_{x, y} \neq 0$, if the interaction of the formerly inactive mode with the main harmonics becomes open by symmetry, its linewidth grows up quickly with the wave number; see the lower TE singlet curve in Fig. 6.

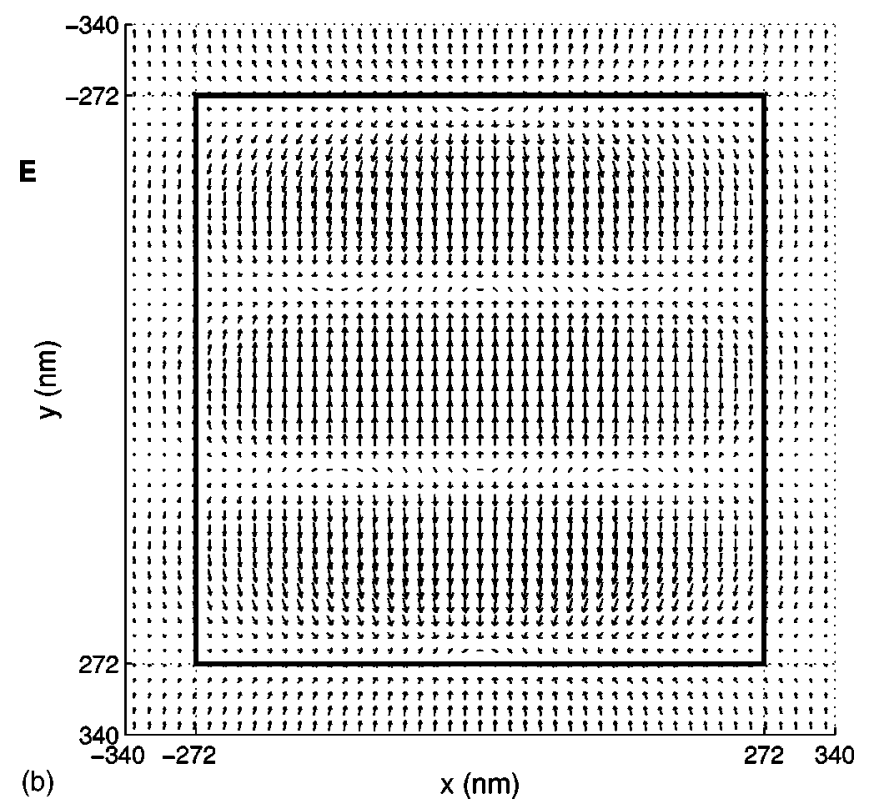

FIG. 9. Electric (a) and magnetic (b) fields in the "slow" TE doublet.

Along the $\Gamma-X$ direction, the lower TE singlet state corresponds to a $B$-type irreducible representation of the point group $C_{1 h}$ (odd over mirror reflection), and the higher TE singlet state corresponds to an $A$-type representation (even). As far as the incoming light is a $B$-type representation in the case of $S$ polarization, the lower singlet state becomes active at $\vartheta \neq 0$ in $S$ polarization. As to the doublet TE state, it splits into a $B$-type fast mode which is active in the $S$ polarization, and an $A$-type mode, active in the $P$ polarization. As to the symmetry properties of the TM quartet, now the magnetic field distributions are assigned as $E, A_{2}$, and $B_{2}$ irreducible representations, whereas the electric field are $E, A_{1}$, and $B_{1}$. Thus the fast singlet and fast doublet components are now $A$ type, which mean that they are optically active in $P$ polar- 
ization. Whereas the slow component of the TM doublet becomes $B$ type in the $\Gamma-X$ direction, and is optically active in the $S$ polarization.

Along the $\Gamma-M$ direction the modes become singlets too. But, as seen in Fig. 6, the corrugation-caused splitting in the model structure is not too strong.

Let us add here that, strictly speaking, anticrossings may take place in the case of crossing bands with the same symmetry. In case of quasiguided modes, however, it is important to remember that the complex energies of modes have to approach each other in order that the effects of anticrossing may take place, not just the real parts of modes energy. For example, there is no anticrossing of slow TE and fast TM doublet components along $\Gamma-X$ in Fig. 6, although they both have the same symmetry $A$. It is because the linewidth of the TE band is approximately one order of magnitude larger than that of the TM band ( $\sim 15$ and $\sim 1.5 \mathrm{meV}$, respectively).

In order to accent the connection between eigenmodes and the position and width of the dips in transmission, in Fig. 5 we emphasized the parts of the transmission spectra between $\Omega(\vartheta)-\gamma(\vartheta) / 2$ and $\Omega(\vartheta)+\gamma(\vartheta) / 2$. First, in Fig. 5 we see that the position and width of all dips, including small ones marked by arrows, correspond directly to $\Omega(\vartheta)$ and $\gamma(\vartheta)$, and it is typical for a situation when the $S$ matrix has poles near the real axis of $\omega .{ }^{13}$ Second, there is a slight asymmetry of dips, which can be attributed to the Fano-type origin of such resonances. Thus slightly asymmetric dips in the transmission may be attributed to the interaction with quasiguided modes, and the measured width of the dips gives information on their radiative lifetime. This opens a direct way to detect such modes experimentally. It is important to distinguish such dips from more asymmetric cusplike Rayleigh anomalies. The latter, however, can be easily identified as diffraction thresholds.

Let us add here that we have considered in this paper the system which is infinite along $(x, y)$ plane, and the light beam cross section is infinite too. This means, e.g., that there are no losses due to the eigenmode propagation along the PCS. However, it is possible to model the finite in-plane size of the PCS and/or beam cross section, assuming the incoming beam to be, e.g., a Gaussian-like convolution of plane waves with different angles of incidence.

To conclude, we have applied a scattering-matrix-based numerical method to calculate the optical transmission properties of a finite-thickness two-dimensionally periodic photonic crystal (photonic crystal slab, PCS). The appearance of well-pronounced dips in the transmissivity of PCS is explained by the excitation of quasi-guided eigenmodes in the slab. We have calculated the energies, linewidths, and electromagnetic fields distributions of the eigenmodes, and analyzed the relationship between their symmetry properties and the optical activity.

\section{ACKNOWLEDGMENTS}

This work was supported in part by CREST, Japan Science and Technology Foundation Corporation, a Grant-inAid for Scientific Research from the Ministry of Education, Science and Culture of Japan, the Russian Foundation for
Basic Research, and the Russian Ministry of Science programs "Nanostructures" and "Information Systems." The authors are thankful to V.V. Popov for drawing their attention to Ref. 30.

\section{APPENDIX A: PLANE-WAVE SOLUTION OF MAXWELL EQUATIONS IN A PERIODICALLY MODULATED SLAB}

In this appendix we solve the Maxwell equations in bulk 1D or 2D periodic photonic crystal (or an infinitely thick PCS), via decomposition into plane waves. This method was described in numerous papers and textbooks; see, e.g., in Ref. 31 and references therein. The most delicate point in such methods is the Fourier transform of the piecewise dielectric function which is poor along the boundaries between dielectrics. Several methods have been proposed to improve the convergence of the planewaves decomposition; see, e.g., Refs. 32 and 33 It appears, however, that for the dielectric structures studied in this paper, the convergency of the direct Fourier decomposition is satisfactory (see below). In the case of metal-based PCS's the convergency appears to be slow, and use of such methods may be very effective.

As shown in Fig. 1, we denote the periodicity plane as $(x, y)$, and the direction of the translational invariance as $\hat{\mathbf{z}}$. In the particular case of $1 \mathrm{D}$ periodicity, we assume the grating planes to be perpendicular to $\hat{\mathbf{x}}$. The dielectric susceptibilities in all constituent materials of the layer are assumed to be local, so that

$$
\mathbf{D}(\mathbf{r})=\int \varepsilon(\mathbf{r}) \delta\left(\mathbf{r}-\mathbf{r}^{\prime}\right) \mathbf{E}\left(\mathbf{r}^{\prime}\right) d \mathbf{r}^{\prime},
$$

where $\varepsilon(\mathbf{r})$ is a periodic function of $x$ and $y$ and does not depend on $z$. Due to the in-plane periodicity, Eq. (A1) is Fourier transformed into

$$
\mathbf{D}_{\mathbf{G}}=\sum_{\mathbf{G}^{\prime}} \varepsilon_{\mathbf{G G}^{\prime}} \mathbf{E}_{\mathbf{G}^{\prime}},
$$

where $\mathbf{D}_{\mathbf{G}}=\left(D_{x, \mathbf{G}}, D_{y, \mathbf{G}}, D_{z, \mathbf{G}}\right)$, etc., $\mathbf{G}=\left(G_{x}, G_{y}, 0\right)$ and $\mathbf{G}^{\prime}$ are reciprocal vectors:

$$
\varepsilon_{\mathbf{G G}^{\prime}}=\frac{1}{S} \int \varepsilon(\mathbf{r}) \exp \left[i\left(\mathbf{G}^{\prime}-\mathbf{G}\right) \mathbf{r}\right] d x d y
$$

(here the integration is performed over the unit cell area $S$ ). An example of such a matrix for the rectangular modulation of $\varepsilon(\mathbf{r})$ used in this paper for numerical calculations is given in Appendix G for completeness. See other examples for, e.g., cylindrical rods in Ref. 31.

In the exact solution, the summation in Eq. (A2) has to be taken over the full reciprocal lattice of vectors $\mathbf{G}^{\prime}$. However, in the numerical calculations the summation should be taken over a finite subset of reciprocal-lattice vectors containing $N_{g}$ elements. For example, in case of 1D periodicity, such a finite subset can be taken as

$$
\mathbf{G}=\left(\frac{2 \pi g}{d_{x}}, 0\right), \quad g=0, \pm 1, \pm 2, \ldots, \pm g_{\max },
$$


$d_{x}$ being the period along $\hat{\mathbf{x}}$. Then the finite subset contains $N_{g}=2 g_{\text {max }}+1$ inverse vectors. In the case of $2 \mathrm{D}$ square periodicity we have taken $N_{g}=\left(2 g_{x, \text { max }}+1\right)\left(2 g_{y \text {, max }}+1\right)$. Numerically, we have estimated $N_{g}$ from the condition that the calculated transmissivity saturates with change of $N_{g} \rightarrow N_{g}$ +1 with an accuracy of the order of $10^{-3}$. For the dielectric structures of the model structure type as in Fig. 1 as well as for a PCS with a polariton pole, ${ }^{17,18}$ this usually occurred at $g_{\max } \approx 6-7$, i.e., $N_{g}=13-15$ in $1 \mathrm{D}$ calculations and 169225 in $2 \mathrm{D}$ calculations; however, we have found that even $g_{\max }=3$ or 4 was already sufficient to understand the main peculiarities of the system behavior in the majority of cases; see an example in Fig. 4.

In what follows we deal with $N_{g^{-}}, 2 N_{g^{-}}$, and $4 N_{g}$-dimensional vectors. The corresponding square matrices will be labeled, as explained in the main text, using usual italic, calligraphic, and open face letters, respectively.

Our goal here is to solve the Maxwell's equation for given input frequency $\omega$, angles of incidence $\varphi$ and $\vartheta$ (azimuthal and polar), and the polarization state. Using the Maxwell's equation

$$
-\frac{1}{c} \frac{\partial \mathbf{H}}{\partial t}=\nabla \times \mathbf{E}
$$

and substituting $(c / i \omega)(\boldsymbol{\nabla} \times \mathbf{E})$ for $\mathbf{H}$ in another Maxwell's equation,

$$
\frac{1}{c} \frac{\partial \mathbf{D}}{\partial t}=\boldsymbol{\nabla} \times \mathbf{H}
$$

we start from the electric-field-resolved Maxwell equation

$$
-\Delta \mathbf{E}+\operatorname{grad} \operatorname{div} \mathbf{E}=\frac{\omega^{2}}{c^{2}} \mathbf{D}
$$

or, in components,

$$
\begin{aligned}
& -\left(\frac{\partial^{2}}{\partial y^{2}}+\frac{\partial^{2}}{\partial z^{2}}\right) E_{x}+\frac{\partial^{2} E_{y}}{\partial x \partial y}+\frac{\partial^{2} E_{z}}{\partial x \partial z}=\frac{\omega^{2}}{c^{2}} D_{x}, \\
& \frac{\partial^{2} E_{x}}{\partial y \partial x}-\left(\frac{\partial^{2}}{\partial z^{2}}+\frac{\partial^{2}}{\partial x^{2}}\right) E_{y}+\frac{\partial^{2} E_{z}}{\partial y \partial z}=\frac{\omega^{2}}{c^{2}} D_{y}, \\
& \frac{\partial^{2} E_{x}}{\partial z \partial x}+\frac{\partial^{2} E_{y}}{\partial z \partial y}-\left(\frac{\partial^{2}}{\partial x^{2}}+\frac{\partial^{2}}{\partial y^{2}}\right) E_{z}=\frac{\omega^{2}}{c^{2}} D_{z} .
\end{aligned}
$$

The solution can be Fourier decomposed into a sum of plane waves of the type

$$
\mathbf{E}(x, y, z, t)=\mathbf{E}_{\mathbf{G}} \exp \left(i k_{x, \mathbf{G}} x+i k_{y, \mathbf{G}} y\right) \times \exp (i K z-i \omega t)
$$

(the same for the induction $\mathbf{D}$ and the magnetic field $\mathbf{H}$ ). Here $k_{x, \mathbf{G}}$ and $k_{y, \mathbf{G}}$ are defined in Eqs. (4), and the eigenvalues of the wave-number $z$ projection $K=K\left(\omega, k_{x}, k_{y}, \mathbf{G}\right)$ to be determined hereafter from the eigenvalue problem.
For the plane wave expansion [Eq. (A11)], the Maxwell equations (A8)-(A10) become a system of linear equations. Equation (A10) takes the form

$$
\sum_{\mathbf{G}^{\prime}} Z_{\mathbf{G G}^{\prime}} E_{z, \mathbf{G}^{\prime}}=K\left[\left(k_{x}+G_{x}\right) E_{x, \mathbf{G}}+\left(k_{y}+G_{y}\right) E_{y, \mathbf{G}}\right],
$$

where the $N_{g} \times N_{g}$ matrix $Z$ is

$$
Z_{\mathbf{G G}^{\prime}}=\left[\left(k_{x}+G_{x}\right)^{2}+\left(k_{y}+G_{y}\right)^{2}\right] \delta_{\mathbf{G G}^{\prime}}-\frac{\omega^{2}}{c^{2}} \varepsilon_{\mathbf{G G}^{\prime}} .
$$

Multiplying Eq. (A12) by the inverse matrix $Z^{-1}$, for each $K$ we obtain

$$
E_{z, \mathbf{G}}=\sum_{\mathbf{G}^{\prime}} Z_{\mathbf{G G}^{\prime}}^{-1} K\left[\left(k_{x}+G_{x}^{\prime}\right) E_{x, \mathbf{G}^{\prime}}+\left(k_{y}+G_{y}^{\prime}\right) E_{y, \mathbf{G}^{\prime}}\right] .
$$

Using Eq. (A14), we can exclude $E_{z, \mathbf{G}}$ from Eqs. (A8) and (A9). Thus we obtain the following system of $2 N_{g}$ linear equations for lateral electric field components $E_{x, \mathbf{G}}$ and $E_{y, \mathbf{G}}$ :

$$
\begin{aligned}
& K^{2} \sum_{\mathbf{G}^{\prime}}\left\{\left[\delta_{\mathbf{G G}^{\prime}}-\left(k_{x}+G_{x}\right) Z_{\mathbf{G G}^{\prime}}^{-1}\left(k_{x}+G_{x}^{\prime}\right)\right] E_{x, \mathbf{G}^{\prime}}\right. \\
&\left.-\left(k_{x}+G_{x}\right) Z_{\mathbf{G G}^{\prime}}^{-1}\left(k_{y}+G_{y}^{\prime}\right) E_{y, \mathbf{G}^{\prime}}\right\} \\
&= \sum_{\mathbf{G}^{\prime}}\left[\frac{\omega^{2}}{c^{2}} \varepsilon_{\mathbf{G G}^{\prime}}-\left(k_{y}+G_{y}\right)^{2} \delta_{\mathbf{G G}^{\prime}}\right] E_{x, \mathbf{G}^{\prime}}+\left(k_{x}\right. \\
&\left.+G_{x}\right)\left(k_{y}+G_{y}\right) E_{y, \mathbf{G}}, \\
& K^{2} \sum_{\mathbf{G}^{\prime}}\left\{-\left(k_{y}+G_{y}\right) Z_{\mathbf{G G}^{\prime}}^{-1}\left(k_{x}+G_{x}^{\prime}\right) E_{x, \mathbf{G}^{\prime}}\right. \\
&\left.+\left[\delta_{\mathbf{G G}^{\prime}}-\left(k_{y}+G_{y}\right) Z_{\mathbf{G G}^{\prime}}^{-1}\left(k_{y}+G_{y}^{\prime}\right)\right] E_{y, \mathbf{G}^{\prime}}\right\} \\
&=\left(k_{x}+G_{x}\right)\left(k_{y}+G_{y}\right) E_{x, \mathbf{G}} \\
&+\sum_{\mathbf{G}^{\prime}}\left[\frac{\omega^{2}}{c^{2}} \varepsilon_{\mathbf{G G}^{\prime}}-\left(k_{x}+G_{x}\right)^{2} \delta_{\mathbf{G G}^{\prime}}\right] E_{y, \mathbf{G}^{\prime}} .
\end{aligned}
$$

Equations (A15) and (A16) can be written in a compact form

$$
\mathcal{M} \overrightarrow{\mathcal{E}}_{\|}=K^{2} \mathcal{N} \overrightarrow{\mathcal{E}}_{\|}
$$

if we introduce $2 N_{g}$ - dimensional vector $\overrightarrow{\mathcal{E}}_{\|}$composed of the lateral components of the electric field, 


$$
\overrightarrow{\mathcal{E}}_{\|}=\left(\begin{array}{c}
E_{x, \mathbf{G}_{1}} \\
\vdots \\
E_{x, \mathbf{G}_{N_{g}}} \\
E_{y, \mathbf{G}_{1}} \\
\vdots \\
E_{y, \mathbf{G}_{N_{g}}}
\end{array}\right),
$$

and $2 N_{g} \times 2 N_{g}$ matrices,

$$
\mathcal{M}=\left(\begin{array}{ll}
M_{11} & M_{12} \\
M_{21} & M_{22}
\end{array}\right), \quad \mathcal{N}=\left(\begin{array}{ll}
N_{11} & N_{12} \\
N_{21} & N_{22}
\end{array}\right)
$$

composed of $N_{g} \times N_{g}$ matrices:

$$
\begin{gathered}
M_{11}=\frac{\omega^{2}}{c^{2}} \varepsilon_{\mathbf{G G}^{\prime}}-\left(k_{y}+G_{y}\right)^{2} \delta_{\mathbf{G G}^{\prime}}, \\
M_{22}=\frac{\omega^{2}}{c^{2}} \varepsilon_{\mathbf{G G}^{\prime}}-\left(k_{x}+G_{x}\right)^{2} \delta_{\mathbf{G G}^{\prime}}, \\
M_{12}=M_{21}=\left(k_{x}+G_{x}\right)\left(k_{y}+G_{y}\right) \delta_{\mathbf{G G}^{\prime}}, \\
N_{11}=\delta_{\mathbf{G G}^{\prime}}-\left(k_{x}+G_{x}\right) Z_{\mathbf{G G}^{\prime}}^{-1}\left(k_{x}+G_{x}^{\prime}\right), \\
N_{22}=\delta_{\mathbf{G G}^{\prime}}-\left(k_{y}+G_{y}\right) Z_{\mathbf{G G}^{\prime}}^{-1}\left(k_{y}+G_{y}^{\prime}\right), \\
N_{12}=N_{21}^{\mathrm{T}}=-\left(k_{x}+G_{x}\right) Z_{\mathbf{G G}^{\prime}}^{-1}\left(k_{y}+G_{y}^{\prime}\right) .
\end{gathered}
$$

In physical problems such as transmission, the frequency $\omega$ is real. It can easily be checked that if all constituent materials of the slab are transparent, $\varepsilon_{\mathbf{G G}^{\prime}}$ is Hermitian. Then $\mathcal{N}$ and $\mathcal{M}$ are Hermitian too. As a result, $K^{2}$ has $2 N_{g}$ real eigenvalues $K_{1}^{2}, K_{2}^{2}, \ldots K_{2 N_{g}}^{2}{ }^{34}$ Thus the plane waves in the decomposition [Eq. (A11)] are either propagating $\left(K^{2}>0\right)$ or exponential $\left(K^{2}<0\right)$. In case of absorptive materials the matrices $\mathcal{N}$ and $\mathcal{M}$ become non-Hermitian, and the eigenvalues of $K^{2}$ are shifted into upper complex half-plane $\operatorname{Im} K^{2} \geqslant 0$ (this corresponds to an exponential decrease of the propagating waves).

For applications it is convenient to write the solution of the eigenproblem [Eq. (A17)] as

$$
(\mathcal{N})^{-1} \mathcal{M} \mathcal{E}_{\|}=\mathcal{E}_{\|} \mathcal{K}^{2}
$$

via two $2 N_{g} \times 2 N_{g}$ matrices: a diagonal matrix

$$
\mathcal{K}=\left(\begin{array}{ccccc}
K_{1} & 0 & 0 & \ldots & 0 \\
0 & K_{2} & 0 & \ldots & 0 \\
\cdots & & & & \\
0 & \ldots & & & K_{2 N_{g}}
\end{array}\right)
$$

having the eigenvalues of the problem [Eq. (A17)] on the diagonal, and a matrix $\mathcal{E}_{\|}$, whose columns are the corresponding eigenvectors

$$
\mathcal{E}_{\|}=\left(\overrightarrow{\mathcal{E}}_{\|}^{(1)}, \overrightarrow{\mathcal{E}}_{\|}^{(2)}, \ldots \overrightarrow{\mathcal{E}}_{\|}^{\left(2 N_{g}\right)}\right)
$$

When calculating $K_{j}=\sqrt{K_{j}^{2}}, j=1,2, \ldots 2 N_{g}$ in Eq. (A23), we fix the cut in such a way that $\operatorname{Re} K_{j} \geqslant 0 .{ }^{27}$ It is important to remember it when discussing the properties of the $\mathrm{S}$ matrix.

\section{APPENDIX B: CALCULATION OF FIELDS, INTERFACE, AND TRANSFER MATRICES}

For a combination of different slabs, we first develop a transfer-matrix formalism. The solution of Maxwell equations in each layer $a$ is decomposed into sets of eigenvectors [see Eq. (A24)], propagating along and opposite to the axis $\hat{\mathbf{z}}$, i.e., $\propto \exp (i K z-i \omega t)$ and $\propto \exp (-i K z-i \omega t)$, respectively. Let us introduce $4 N_{g}$-dimensional amplitudes of partial waves in each plane $z$,

$$
\overrightarrow{\mathrm{A}}(z)=\left(\begin{array}{c}
\overrightarrow{\mathcal{A}}^{+}(z) \\
\overrightarrow{\mathcal{A}}^{-}(z)
\end{array}\right),
$$

where $\overrightarrow{\mathcal{A}}_{j}^{ \pm}(z)$ are $2 N_{g}$-dimensional amplitudes of plane waves, propagating, respectively, along and counter to the axis $\overrightarrow{\mathbf{z}}$, at $z$. Let us define the $4 N_{g}$-dimensional amplitude $\vec{A}$ in such a way that the $2 N_{g}$-dimensional vector of in-plane electric-field components [Eq. (A24)] at $z$ is the matrix product of a matrix $\left(\mathcal{E}_{\|}, \mathcal{E}_{\|}\right)$[with $4 N_{g}$ columns and $2 N_{g}$ rows] and $\overrightarrow{\mathbb{A}}$ :

$$
\overrightarrow{\mathcal{E}}_{\|}(z)=\left(\mathcal{E}_{\|}, \mathcal{E}_{\|}\right) \overrightarrow{\mathrm{A}}(z)
$$

This point is the central one for the formulation of the transfer-matrix formalism; it becomes possible because the $2 N_{g} \times 2 N_{g}$-dimensional matrix $\mathcal{E}_{\|}$does not depend on $z$, but depends on the material parameters of the current layer.

Transfer matrices $\mathrm{T}$ connect the amplitudes at different planes $z$ and $z^{\prime}$, and, thus, they are $4 N_{g} \times 4 N_{g}$ matrices. The simplest task is to construct the transfer matrix $\mathrm{T}_{L}$ over a distance $L$ (along $\hat{\mathbf{z}}$ ) inside the same layer. It is a matrix which transfers the vector of amplitudes from plane $z$ to $z+L$,

$$
\overrightarrow{\mathrm{A}}(z+L)=\mathrm{T}_{L} \overrightarrow{\mathrm{A}}(z),
$$

and it can be written as a diagonal matrix,

$$
\mathrm{T}_{L}=\left(\begin{array}{cc}
\exp [i \mathcal{K} L] & 0 \\
0 & \exp [-i \mathcal{K} L]
\end{array}\right),
$$

where the matrix exponents are diagonal matrices with $\exp \left( \pm K_{j} L\right), j=0,1 \ldots 2 N_{g}$ on the main diagonal.

To construct the interface matrix $\mathbb{T}_{b, a}$ through an interface from slab $a$ to slab $b$, we can use the continuity conditions of tangential ( $x, y$ in our case) components of the electric and magnetic fields at the interface. There are $4 N_{g}$ tangential components of $\vec{E}_{x}, \vec{E}_{y}, \vec{H}_{x}, \vec{H}_{y}$ (grouped into a pair of $2 N_{g}$-dimensional vectors $\overrightarrow{\mathcal{E}}_{\|}$and $\left.\overrightarrow{\mathcal{H}}_{\|}\right)$, and $4 N_{g}$ continuity conditions. It can be shown easily that other Maxwell bound- 
ary conditions (on normal components $\vec{D}_{z}, \vec{H}_{z}$ ) follow from these conditions. Note that this amount, $4 N_{g}$, matches exactly the dimensionality of the amplitude vector $\overrightarrow{\mathrm{A}}$.

Let us define a material matrix $\mathbb{F}_{j}, j=a, b$, which converts the vector of amplitudes at fixed $z$ to the hypervector of in-plane components of electric and magnetic fields in this point,

$$
\left(\begin{array}{c}
\overrightarrow{\mathcal{E}}_{\|}(z) \\
\overrightarrow{\mathcal{H}}_{\|}(z)
\end{array}\right)=\mathbb{F} \vec{A}(z)
$$

where the hypervectors $\overrightarrow{\mathcal{E}}_{\|}$are defined in Eq. (A18) (the same type of columns are used as for $\overrightarrow{\mathcal{H}}_{\|}$, but they are composed of $x$ and $y$ components of magnetic fields). Then, from the continuity of the tangential components at the interface at $z$ $=z_{b, a}$, we obtain

$$
\left.\overrightarrow{\mathrm{A}}\right|_{z=z_{b, a}+0}=\left.\mathbb{T}_{b, a} \overrightarrow{\mathrm{A}}\right|_{z=z_{b, a}-0},
$$

with the interface matrix

$$
\mathbb{T}_{b, a}=\mathbb{F}_{b}^{-1} \mathbb{F}_{a} .
$$

In order to find the matrix $\mathbb{F}$ for each slab, let us use Eq. (B2) and the analogous equation for $\overrightarrow{\mathcal{H}}_{\|}$. For each partial plane wave it follows from Eq. (A5) that

$$
\begin{aligned}
H_{x, \mathbf{G}}= & \frac{K c}{\omega} \sum_{\mathbf{G}^{\prime}}\left\{\left(k_{y}+G_{y}\right) Z_{\mathbf{G G}^{\prime}}^{-1}\left(k_{x}+G_{x}^{\prime}\right) E_{x, \mathbf{G}^{\prime}}+\left[\left(k_{y}\right.\right.\right. \\
& \left.\left.\left.+G_{y}\right) Z_{\mathbf{G G}^{\prime}}^{-1}\left(k_{y}+G_{y}^{\prime}\right)-\delta_{\mathbf{G G}^{\prime}}\right] E_{y, \mathbf{G}^{\prime}}\right\}, \\
H_{y, \mathbf{G}}= & \frac{K c}{\omega} \sum_{\mathbf{G}^{\prime}}\left\{\left[\delta_{\mathbf{G G}^{\prime}}-\left(k_{x}+G_{x}\right) Z_{\mathbf{G G}^{\prime}}^{-1}\left(k_{x}+G_{x}^{\prime}\right)\right] E_{x, \mathbf{G}^{\prime}}-\left(k_{x}\right.\right. \\
& \left.\left.+G_{x}\right) Z_{\mathbf{G G}^{\prime}}^{-1}\left(k_{y}+G_{y}^{\prime}\right) E_{y, \mathbf{G}^{\prime}}\right\} .
\end{aligned}
$$

Introducing a $2 N_{g} \times 2 N_{g}$ matrix

$$
\mathcal{C}=\left(\begin{array}{cc}
\left(k_{y}+G_{y}\right) Z_{\mathbf{G G}^{\prime}}^{-1}\left(k_{x}+G_{x}^{\prime}\right) & \left(k_{y}+G_{y}\right) Z_{\mathbf{G G}^{\prime}}^{-1}\left(k_{y}+G_{y}^{\prime}\right)-\delta_{\mathbf{G G}^{\prime}} \\
\delta_{\mathbf{G G}^{\prime}}-\left(k_{x}+G_{x}\right) Z_{\mathbf{G G}^{\prime}}^{-1}\left(k_{x}+G_{x}^{\prime}\right) & -\left(k_{x}+G_{x}\right) Z_{\mathbf{G G}^{\prime}}^{-1}\left(k_{y}+G_{y}^{\prime}\right)
\end{array}\right)=\left(\begin{array}{cc}
-N_{21} & -N_{22} \\
N_{11} & N_{12}
\end{array}\right),
$$

we come to the conclusion that

$$
\overrightarrow{\mathcal{H}}_{\|}=\frac{c}{\omega}\left(\mathcal{C} \mathcal{E}_{\|} \mathcal{K},-\mathcal{C} \mathcal{E}_{\|} \mathcal{K}\right) \overrightarrow{\mathrm{A}}
$$

Thus the material matrix is

$$
\mathbb{F}=\left(\begin{array}{cc}
\mathcal{E}_{\|} & \mathcal{E}_{\|} \\
\frac{c}{\omega} \mathcal{C} \mathcal{E}_{\|} \mathcal{K} & -\frac{c}{\omega} \mathcal{C} \mathcal{E}_{\|} \mathcal{K}
\end{array}\right) .
$$

\section{APPENDIX C: INPUT AMPLITUDES}

In order to close the transfer-matrix formalism, we have to define the input amplitudes [Eq. (11)] and the scattering condition. Let us reserve the top position in each $N_{g}$-dimensional vector of $E_{x, \mathbf{G}}$, etc., type for the $G=0$ main harmonic. Then, for the geometry of light incidence from the vacuum side specified in Fig. 1 and the Eq. (1) case of $S$ polarization [vector $\mathbf{E}$ parallel to the $(x, y)$ plane], we obtain

$$
\left.\overrightarrow{\mathcal{A}}_{\mathrm{v}, S}^{+} \equiv \overrightarrow{\mathcal{A}}_{\mathrm{v}, S}^{+}(z)\right|_{z=-0}=\left(\begin{array}{c}
\sin \varphi \\
0 \\
\vdots \\
-\cos \varphi \\
0 \\
\vdots
\end{array}\right)
$$

[the only nonzero components are the first and the $\left(N_{g}+1\right)$ th]. In the case of $P$ polarization we obtain

$$
\left.\overrightarrow{\mathcal{A}}_{\mathrm{v}, P}^{+} \equiv \overrightarrow{\mathcal{A}}_{\mathrm{v}, P}^{+}(z)\right|_{z=-0}=\left(\begin{array}{c}
\cos \varphi \cos \vartheta \\
0 \\
\vdots \\
\sin \varphi \cos \vartheta \\
0 \\
\vdots
\end{array}\right) .
$$

As to the scattering condition, it is understood that the amplitudes of the counter-propagating waves on the substrate side of the system $\left(z=L_{z}+0\right)$ are just zeros in any polarization: 


$$
\left.\overrightarrow{\mathcal{A}}_{\mathrm{s}}^{-} \equiv \overrightarrow{\mathcal{A}}_{\mathrm{s}}^{-}(z)\right|_{z=L_{z}+0}=\left(\begin{array}{c}
0 \\
\vdots \\
0
\end{array}\right) .
$$

\section{APPENDIX D: FROM TRANSFER- TO SCATTERING- MATRIX FORMALISM}

Now we are ready to close the transfer-matrix formalism: the total transfer matrix through the whole system can be calculated as

$$
\overrightarrow{\mathrm{A}}_{\mathrm{s}}=\mathbb{T}_{\text {tot }} \overrightarrow{\mathrm{A}}_{\mathrm{v}} \text {, }
$$

with

$$
\mathbb{T}_{\text {tot }}=\mathbb{T}_{\mathrm{s}, a_{N}} \mathrm{~T}_{L_{N}}^{\left(a_{N}\right)} \mathbb{T}_{a_{N}, a_{N-1}} \ldots \mathbb{T}_{L_{1}}^{\left(a_{1}\right)} \mathrm{T}_{a_{1}, \mathrm{v}},
$$

where the system is supposed to contain $N$ different slabs $a_{j}$, $j=1, \ldots N(N=1$ for our model structure in Fig. 1).

However, as it was first discussed by Ko and Inkson, ${ }^{23}$ the inevitable existence of evanescent solutions (with $K^{2}<0$ ), makes this transfer-matrix calculation unstable very quickly. This difficulty can be easily understood from the definition of the transfer matrix $T_{L}$ [Eq. (B4)]: for evanescent eigenvalues, the lower diagonal block of this matrix contains exponentially large components.

In order to avoid this disadvantage, a scattering matrix formalism was proposed, ${ }^{23}$ which avoids this problem by means of rearranging the components of amplitudes. Within the scattering-matrix method, instead of a description of the system via a combination of amplitudes of along- and counter-propagating waves [Eq. (B1)], a scattering matrix $\mathrm{S}_{0, N+1}$ is introduced, which transfers the input amplitudes

$$
\overrightarrow{\mathrm{B}}_{\mathrm{in}}=\left(\begin{array}{c}
\overrightarrow{\mathcal{A}}_{\mathrm{v}}^{+} \\
\overrightarrow{\mathcal{A}}_{\mathrm{s}}^{-}
\end{array}\right)
$$

into output ones,

$$
\overrightarrow{\mathrm{B}}_{\text {out }}=\left(\begin{array}{c}
\overrightarrow{\mathcal{A}}_{\mathrm{s}}^{+} \\
\overrightarrow{\mathcal{A}}_{\mathrm{v}}^{-}
\end{array}\right),
$$

and is coupled by the total scattering matrix

$$
\overrightarrow{\mathrm{B}}_{\mathrm{out}}=\mathrm{S}_{\mathrm{v}, \mathrm{s}} \overrightarrow{\mathrm{B}}_{\mathrm{in}} \text {. }
$$

The total scattering matrix can be calculated iteratively, ${ }^{23}$ starting from an obvious condition

$$
\mathrm{S}_{\mathrm{v}, \mathrm{v}}=\mathrm{I} \text {, }
$$

where $\mathbb{I}$ is $4 N_{g} \times 4 N_{g}$ unit matrix, and using the following procedure to calculate the scattering matrix $\mathrm{S}_{M+1}$ of a system containing $M+1$ layers if we know the total scattering matrix $\mathrm{S}_{M}$ of the system with $M$ layers as well as the inverse transfer matrix $\mathrm{T}$ through the additional $(M+1)$ th layer,

$$
\mathrm{S}_{M} \equiv\left(\begin{array}{ll}
\mathcal{S}_{11} & \mathcal{S}_{12} \\
\mathcal{S}_{21} & \mathcal{S}_{22}
\end{array}\right)
$$

and

$$
\mathrm{T} \equiv\left(\begin{array}{ll}
\mathcal{T}_{11} & \mathcal{T}_{12} \\
\mathcal{T}_{21} & \mathcal{T}_{22}
\end{array}\right)
$$

For example, if we add an interface with interface matrix $\mathbb{T}_{b, a}$ [Eq. (B7)], then $\mathbb{T}=\mathbb{T}_{a, b}=\mathbb{T}_{b, a}^{-1}$. If we add a slab $L$ of same material, $\mathrm{T}=\mathbb{T}_{-L}$.

Then

$$
\mathrm{S}_{M+1}=\left(\begin{array}{cc}
\mathcal{D} \mathcal{S}_{11} & \mathcal{D E} \\
\mathcal{S}_{21}+\mathcal{S}_{22} \mathcal{T}_{21} \mathcal{D} \mathcal{S}_{11} & \mathcal{S}_{22} \mathcal{T}_{21} \mathcal{D E}+\mathcal{S}_{22} \mathcal{T}_{22}
\end{array}\right),
$$

where

$$
\mathcal{E}=\left(\mathcal{S}_{12} \mathcal{T}_{22}-\mathcal{T}_{12}\right), \quad \mathcal{D}=\left(\mathcal{T}_{11}-\mathcal{S}_{12} \mathcal{T}_{21}\right)^{-1}
$$

If we add an $L$-thick layer of type $j$, from Eq. (D9) we obtain

$$
\mathrm{S}_{M+1}=\left(\begin{array}{cc}
\exp \left[i \mathcal{K}^{(j)} L\right] & \mathcal{O} \\
\mathcal{O} & \mathcal{I}
\end{array}\right) \mathrm{S}_{M}\left(\begin{array}{cc}
\mathcal{I} & \mathcal{O} \\
\mathcal{O} & \exp \left[i \mathcal{K}^{(j)} L\right]
\end{array}\right)
$$

where $\mathcal{I}$ and $\mathcal{O}$ are $2 N_{g} \times 2 N_{g}$ unit and zero matrices, respectively. From Eq. (D11) it is seen that, indeed, the growing exponents (for increscent waves), $\exp \left[-i \mathcal{K}^{(j)} L\right]$, do not appear in the scattering matrix formalism.

\section{APPENDIX E: CALCULATION OF TRANSMISSION, REFLECTION, DEFLECTION, AND ABSORPTION}

Knowledge of the scattering matrix $\mathrm{S}$ of the whole system and of the input amplitude vector $\vec{B}_{\text {in }}$ allows us to calculate the reflection, transmission and absorption coefficients. The simplest way to do this is to calculate the $z$ projections of the Poynting vector for incoming, reflected, transmitted, and deflected (into vacuum and substrate) waves,

$$
\begin{aligned}
P^{(\mathrm{in})}= & \frac{c}{16 \pi}\left[\left(E_{x, 0, \mathrm{v}}^{+}\right) * H_{y, 0, \mathrm{v}}^{+}+E_{x, 0, \mathrm{v}}^{+}\left(H_{y, 0, \mathrm{v}}^{+}\right) *-\left(E_{y, 0, \mathrm{v}}^{+}\right) * H_{x, 0, \mathrm{v}}^{+}\right. \\
& \left.-E_{y, 0, \mathrm{v}}^{+}\left(H_{x, 0, \mathrm{v}}^{+}\right) *\right], \\
P^{(\mathrm{r})}= & \frac{c}{16 \pi}\left[\left(E_{x, 0, \mathrm{v}}^{-}\right) * H_{y, 0, \mathrm{v}}^{-}+E_{x, 0, \mathrm{v}}^{-}\left(H_{y, 0, \mathrm{v}}^{-} *-\left(E_{y, 0, \mathrm{v}}^{-} * H_{x, 0, \mathrm{v}}^{-}\right.\right.\right. \\
& \left.-E_{y, 0, \mathrm{v}}^{-}\left(H_{x, 0, \mathrm{v}}^{-}\right) *\right], \\
P^{(\mathrm{t})}= & \frac{c}{16 \pi}\left[\left(E_{x, 0, \mathrm{~s}}^{+}\right) * H_{y, 0, \mathrm{~s}}^{+}+E_{x, 0, \mathrm{~s}}^{+}\left(H_{y, 0, \mathrm{~s}}^{+} *-\left(E_{y, 0, \mathrm{~s}}^{+}\right) * H_{x, 0, \mathrm{~s}}^{+}\right.\right. \\
& \left.-E_{y, 0, \mathrm{~s}}^{+}\left(H_{x, 0, \mathrm{~s}}^{+}\right) *\right], \\
P^{(\mathrm{d}, \mathrm{v})}= & \frac{c}{16 \pi} \sum_{\mathbf{G} \neq 0}\left[\left(\left(E_{x, \mathbf{G}, \mathrm{v}}^{-}\right) * H_{y, \mathbf{G}, \mathrm{v}}^{-}+E_{x, \mathbf{G}, \mathrm{v}}^{-}\left(H_{y, \mathbf{G}, \mathrm{v}}^{-}\right) *\right.\right. \\
& \left.-\left(E_{y, \mathbf{G}, \mathrm{v}}^{-}\right) * H_{x, \mathbf{G}, \mathrm{v}}^{-}-E_{y, \mathbf{G}, \mathrm{v}}^{-}\left(H_{x, \mathbf{G}, \mathrm{v}}^{-}\right) *\right],
\end{aligned}
$$




$$
\begin{aligned}
P^{(\mathrm{d}, \mathrm{s})}= & \frac{c}{16 \pi} \sum_{\mathbf{G} \neq 0}\left[\left(\left(E_{x, \mathbf{G}, \mathrm{s}}^{+}\right) * H_{y, \mathbf{G}, \mathrm{s}}^{+}+E_{x, \mathbf{G}, \mathrm{s}}^{+}\left(H_{y, \mathbf{G}, \mathrm{s}}^{+}\right) *\right.\right. \\
& \left.-\left(E_{y, \mathbf{G}, \mathrm{s}}^{+}\right) * H_{x, \mathbf{G}, \mathrm{s}}^{+}-E_{y, \mathbf{G}, \mathrm{s}}^{+}\left(H_{x, \mathbf{G}, \mathrm{s}}^{+}\right) *\right],
\end{aligned}
$$

and the components of electric and magnetic fields are calculated via Eq. (B5). Then the reflection, transmission, and deflection coefficients can be calculated as shown in Eq. (13), note that $P^{(\mathrm{r})}$ and $P^{(\mathrm{d}, \mathrm{v})}$ entering this equation with a negative sign. The absorption coefficient is

$$
A=1-R-T-D \text {. }
$$

$A=0$ in a system without dissipation. In case of dissipation, $A \neq 0$, and the frequency dependence of this coefficient, via the fluctuation-dissipation theorem, is connected to the emission.

\section{APPENDIX F: CASE OF SPATIALLY UNIFORM SLABS}

In Appendix F we illustrate the general solution given in Appendix A on the simplest case of spatially uniform slabs. The dielectric matrix [Eq. (A3)] is diagonal:

$$
\varepsilon_{\mathbf{G G}^{\prime}}=\varepsilon \delta_{\mathbf{G G}^{\prime}} .
$$

Instead of Eq. (A13) we have

$$
\begin{gathered}
Z_{\mathbf{G G}^{\prime}}=\left(\begin{array}{cc}
k_{x, \mathbf{G}}^{2}+k_{y, \mathbf{G}}^{2}-\frac{\omega^{2} \varepsilon}{c^{2}}
\end{array}\right) \delta_{\mathbf{G G}^{\prime}}, \\
\mathcal{M}=\left(\begin{array}{cc}
\frac{\omega^{2} \varepsilon}{c^{2}}-k_{y, \mathbf{G}}^{2} & k_{x, \mathbf{G}} k_{y, \mathbf{G}} \\
k_{x, \mathbf{G}} k_{y, \mathbf{G}} \frac{\omega^{2} \varepsilon}{c^{2}}-k_{x, \mathbf{G}}^{2}
\end{array}\right) \delta_{\mathbf{G G}^{\prime},}(\mathrm{F} 3) \\
k_{x, \mathbf{G}}^{2}+k_{y, \mathbf{G}}^{2}-\frac{\omega^{2} \varepsilon}{c^{2}}
\end{gathered}\left(\begin{array}{cc}
k_{y, \mathbf{G}}^{2}-\frac{\omega^{2} \varepsilon}{c^{2}} & -k_{x, \mathbf{G}} k_{y, \mathbf{G}} \\
-k_{x, \mathbf{G}} k_{y, \mathbf{G}} & k_{x, \mathbf{G}}^{2}-\frac{\omega^{2} \varepsilon}{c^{2}}
\end{array}\right) \delta_{\mathbf{G G}^{\prime},}
$$

and

$$
\mathcal{N}^{-1} \mathcal{M}=\left(\frac{\omega^{2} \varepsilon}{c^{2}}-k_{x, \mathbf{G}}^{2}-k_{y, \mathbf{G}}^{2}\right) \Delta_{\mathbf{G G}^{\prime}}
$$

where

$$
\Delta_{\mathbf{G G}^{\prime}}=\left(\begin{array}{cc}
1 & 0 \\
0 & 1
\end{array}\right) \delta_{\mathbf{G G}^{\prime}} .
$$

Instead of Eq. (A17) we obtain

$$
\left(\frac{\omega^{2} \varepsilon}{c^{2}}-k_{x, \mathbf{G}}^{2}-k_{y, \mathbf{G}}^{2}\right) \Delta_{\mathbf{G G}^{\prime}} \overrightarrow{\mathcal{E}}_{\|}=K^{2} \overrightarrow{\mathcal{E}}_{\|} .
$$

Thus, for $P$ and $S$ polarizations [see Eq. (A24)] in one dimension, we obtain

$$
\mathcal{E}_{\|}=\Delta_{\mathbf{G G}^{\prime}}
$$

and degenerate eigenvalues

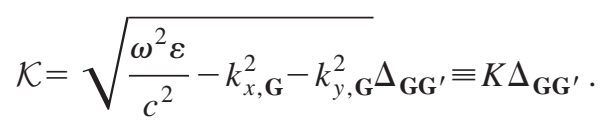

Instead of Eq. (A14), and because $K=k_{z, \mathbf{G}}$, we obtain

$$
E_{z, \mathbf{G}}=-\frac{k_{x, \mathbf{G}} E_{x, \mathbf{G}}+k_{y, \mathbf{G}} E_{y, \mathbf{G}}}{k_{z, \mathbf{G}}},
$$

which is nothing but the condition of the transverse wave for each harmonic $\mathbf{k}_{\mathbf{G}} \mathbf{E}_{\mathbf{G}}=0$, where $\mathbf{k}_{\mathbf{G}}=\left(k_{x, \mathbf{G}}, k_{y, \mathbf{G}}, k_{z, \mathbf{G}}\right)$ is the light wave vector inside the homogeneous layer.

\section{APPENDIX G: DIELECTRIC SUSCEPTIBILITY MATRIX FOR 1D AND 2D RECTANGULAR PERIODICALLY PATTERNED LAYER}

For self-consistency, here we give the well-known formulas for $\varepsilon_{\mathbf{G G}^{\prime}}$ for a rectangular-type modulation of the dielectric constant in the PCS. If the 1D patterned layer consists of $L_{x^{-}}$and $\left(d_{x}-L_{x}\right)$-wide regions with dielectric susceptibilities $\varepsilon_{2}$ and $\varepsilon_{1}$, respectively, the dielectric matrix $\varepsilon_{\mathbf{G G}^{\prime}}$ [Eq. (A3)] is

$$
\varepsilon_{g g^{\prime}}=\varepsilon_{1} \delta_{g g^{\prime}}+\left(\varepsilon_{2}-\varepsilon_{1}\right) \frac{\sin \left[\pi\left(g-g^{\prime}\right) \frac{L_{x}}{d_{x}}\right]}{\pi\left(g-g^{\prime}\right)} .
$$

In the case of $2 \mathrm{D}$ rectangular modulation (as in Fig. 1), we have

$$
\begin{aligned}
\varepsilon_{\mathbf{G G}^{\prime}}= & \varepsilon_{1} \delta_{\mathbf{G G}^{\prime}}+\left(\varepsilon_{2}-\varepsilon_{1}\right) \\
\times & \frac{\sin \left[\pi\left(g_{x}-g_{x}^{\prime}\right) \frac{L_{x}}{d_{x}}\right] \sin \left[\pi\left(g_{y}-g_{y}^{\prime}\right) \frac{L_{y}}{d_{y}}\right]}{\pi^{2}\left(g_{x}-g_{x}^{\prime}\right)\left(g_{y}-g_{y}^{\prime}\right)} .
\end{aligned}
$$

${ }^{1}$ A. A. Maradudin and A. R. McGurn, J. Opt. Soc. Am. B 10, 307 (1993).

${ }^{2}$ D. M. Atkin, P. S. J. Russell, T. A. Birks, and P. J. Roberts, J. Chem. Phys. 43, 1035 (1996).

${ }^{3}$ A. Sentenac, J.-J. Greffet, and F. Pincemin, J. Opt. Soc. Am. B 14, 339 (1997).
${ }^{4}$ D. Labilloy, H. Benisty, C. Weisbuch, C. J. M. Smith, T. F. Krauss, R. Houdré, and U. Oesterle, Phys. Rev. B 59, 1649 (1999).

${ }^{5}$ S. G. Johnson, S. Fan, P. R. Villeneuve, J. D. Joannopoulos, and L. A. Kolodziejski, Phys. Rev. B 60, 5751 (1999).

${ }^{6}$ V. N. Astratov, D. M. Whittaker, I. S. Culshaw, R. M. Stevenson, 
M. S. Skolnick, T. F. Krauss, and R. M. De La Rue, Phys. Rev. B 60, R16 255 (1999).

${ }^{7}$ D. M. Whittaker and I. S. Culshaw, Phys. Rev. B 60, 2610 (1999).

${ }^{8}$ L. Wendler and T. Kraft, Physica B 271, 33 (1999).

${ }^{9}$ P. Paddon and J. F. Young, Phys. Rev. B 61, 2090 (2000).

${ }^{10}$ T. Ochiai and K. Sakoda, Phys. Rev. B 63, 125107 (2001).

${ }^{11}$ T. Ochiai and K. Sakoda, Phys. Rev. B 64, 045108 (2001).

${ }^{12}$ N. Carlsson, T. Takemori, K. Asakawa, and Y. Katayama, J. Opt. Soc. Am. B 18, 1260 (2001).

${ }^{13} \mathrm{M}$. Nevière, in Electromagnetic Theory of Gratings, edited by R. Petit (Springer-Verlag, Berlin, 1980), Chap. 5, pp. 123-157.

${ }^{14}$ R. W. Wood, Philos. Mag. 4, 396 (1902).

${ }^{15}$ J. W. S. Rayleigh, Philos. Mag. 14, 60 (1907).

${ }^{16}$ U. Fano, Phys. Rev. 124, 1866 (1961).

${ }^{17}$ A. L. Yablonskii, E. A. Muljarov, N. A. Gippius, S. G. Tikhodeev, T. Fujita, and T. Ishihara, J. Phys. Soc. Jpn. 70, 1137 (2001).

${ }^{18}$ R. Shimada, A. L. Yablonskii, S. G. Tikhodeev, and T. Ishihara, IEEE J. Quantum Electron. (to be published).

${ }^{19}$ A. L. Yablonskii, E. A. Muljarov, N. A. Gippius, S. G. Tikhodeev, and T. Ishihara, Phys. Status Solidi B (to be published).

${ }^{20}$ T. Fujita, Y. Sato, T. Kuitani, and T. Ishihara, Phys. Rev. B 57, 12 428 (1998).

${ }^{21}$ P. S. J. Russel, D. Atkin, T. A. Birks, and P. J. Roberts, in Microcavities and Photonic Bandgaps, edited by J. Rarity and C. Weisbuch (Kluwer, Dordrecht, 1996), pp. 203-218.

${ }^{22}$ O. Painter, R. K. Lee, A. Scherer, A. Yariv, J. D. O'Brien, P. D. Dapkus, and I. Kim, Science 284, 1819 (1999).

${ }^{23}$ D. Y. K. Ko and J. C. Inkson, Phys. Rev. B 38, 9945 (1988).

${ }^{24}$ P. Lalanne and H. Benisty, J. Appl. Phys. 89, 1512 (2001).

${ }^{25}$ L. Pilozzi, A. D’Andrea, and R. D. Sole, Phys. Rev. B 54, 10751 (1996).
${ }^{26}$ T. Ishihara, T. Fujita, A. Seki, and H. Nakashima, Mol. Cryst. Liq. Cryst. 371, 167 (2001).

${ }^{27}$ For the discussions here, it is important to fix the rule of calculating the square root. As in most software packages, we assume that $\operatorname{Re} \sqrt{A} \geqslant 0$ for any complex A, and $\operatorname{Im} \sqrt{B}>0$ for $\operatorname{Im} B=0$ and $\operatorname{Re} B<0$.

${ }^{28}$ A. Yariv, Quantum Electronics (Wiley, New York, 1989).

${ }^{29}$ A. I. Baz', Ya. B. Zel'dovich, and A. M. Perelomov, Scattering, Reactions and Decay in Nonrelativistic Quantum Mechanics (Nauka Moscow, 1966) (in Russian) [English translation by Z. Lerman: Jerusalem, Israel Program for Scientific Translations, available from the U.S. Dept. of Commerce, Clearinghouse for Federal Scientific and Technical Information, Springfield, VA, 1969].

${ }^{30}$ P. Lampariello, F. Frezza, and A. A. Oliner, IEEE Trans. Microwave Theory Tech. 38, 1831 (1990).

${ }^{31}$ K. Sakoda, Optical Properties of Photonic Crystals (Springer, New York, 2001).

${ }^{32}$ R. D. Meade, A. M. Rappe, K. D. Brommer, J. D. Joannopoulos, and O. L. Alerhand, Phys. Rev. B 48, 8434 (1993).

${ }^{33}$ E. Popov and M. Nevière, J. Opt. Soc. Am. A 17, 1773 (2000).

${ }^{34}$ For Hermitian $\mathcal{M}$ and $\mathcal{N}$, it can be shown that for any two eigenvalues $K_{n}^{2}$ and $K_{m}^{2}$ of the linear problem [Eq. (A17)], $\left(K_{n}^{2}\right.$ $\left.-K_{m}^{2 \dagger}\right)\left\{\overrightarrow{\mathcal{E}}_{\|}^{(n) \dagger} \mathcal{N} \overrightarrow{\mathcal{E}}_{\|}^{(m)}\right\}=0$. Because of Eqs. (B10) and (B11), $\overrightarrow{\mathcal{E}}_{\|}^{(n) \dagger} \mathcal{N} \overrightarrow{\mathcal{E}}_{\|}^{(m)} \propto \overrightarrow{\mathcal{E}}_{\|}^{(n) \dagger} \times \overrightarrow{\mathcal{H}}_{\|}^{(m)}=\Sigma_{\mathbf{G}}\left[E_{x, \mathbf{G}}^{(m) \dagger} H_{y, \mathbf{G}}^{(n)}-E_{y, \mathbf{G}}^{(m) \dagger} H_{x, \mathbf{G}}^{(n)}\right] . \quad$ Thus, for $m=n$ we obtain that the eigenvalues are real, $K_{m}^{2}=K_{m}^{2 \dagger}$, and for $m \neq n$ we obtain the condition of orthogonality $\overrightarrow{\mathcal{E}}_{\|}^{(n) \dagger} \times \overrightarrow{\mathcal{H}}_{\|}^{(m)}$ $=0$, which replaces the standard orthogonality condition in case of electrodynamics; see, e.g., [A. W. Snyder and J. D. Love, Optical Waveguide Theory (Kluwer, Dordrecht, 1983)]. 